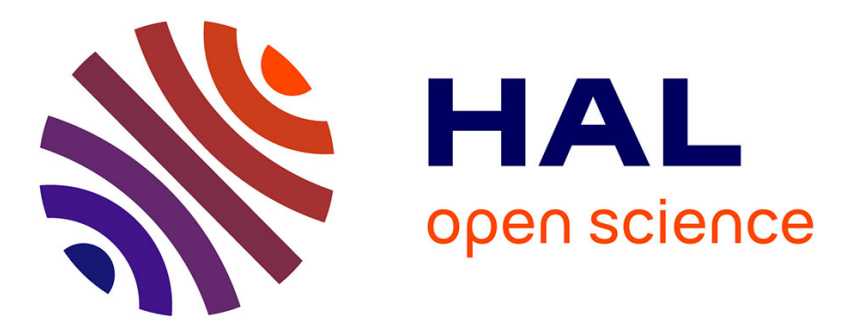

\title{
Sensitivity of the 1D shallow water equations with source terms: Solution method for discontinuous flow
}

Carole Delenne, Pascal Finaud-Guyot, Vincent Guinot, Bernard Cappelaere

\section{To cite this version:}

Carole Delenne, Pascal Finaud-Guyot, Vincent Guinot, Bernard Cappelaere. Sensitivity of the 1D shallow water equations with source terms: Solution method for discontinuous flow. International Journal for Numerical Methods in Fluids, 2010, pp.10.1002/fld.2398. 10.1002/fld.2398 . hal00515826

\section{HAL Id: hal-00515826 \\ https://hal.science/hal-00515826}

Submitted on 25 Nov 2020

HAL is a multi-disciplinary open access archive for the deposit and dissemination of scientific research documents, whether they are published or not. The documents may come from teaching and research institutions in France or abroad, or from public or private research centers.
L'archive ouverte pluridisciplinaire HAL, est destinée au dépôt et à la diffusion de documents scientifiques de niveau recherche, publiés ou non, émanant des établissements d'enseignement et de recherche français ou étrangers, des laboratoires publics ou privés. 


\title{
Sensitivity of the 1D shallow water equations with source terms: Solution method for discontinuous flows
}

\author{
C. Delenne, P. Finaud-Guyot, V. Guinot and B. Cappelaere
}

24th June 2010

\begin{abstract}
A finite volume-based numerical technique is presented concerning the sensitivity of the solution of the one-dimensional Shallow Water Equations (SWEs) with scalar transport. An approximate Riemann solver is proposed for direct sensitivity calculation even in the presence of discontinuous solutions. The Shallow Water Sensitivity Equations (SWSEs) are first derived as well as the expressions of the sensitivity source terms, initial and boundary conditions. The numerical technique is then detailed and application examples are provided to assess the method's efficiency in estimating the sensitivity to different parameters (friction coefficient and initial and boundary conditions). The application of the dam-break problem to a trapezoidal channel is also provided. The comparison with the analytical solution and the classical empirical approach illustrates the usefulness of the direct sensitivity calculation.

Keywords: shallow water equations; sensitivity; finite volume; Riemann solver; source terms; initial and boundary conditions.
\end{abstract}

\section{Introduction}

The assessment of the sensitivity of a model output to different parameters is a key step in the optimization of model performance, since it can be used to define the relevant parameters for the calibration procedures. Sensitivity analysis is thus seen by some authors [15] as a prerequisite for model building and exploitation, in any scientific discipline where models are used. In shallow water modelling, sensitivity analysis may be used to determine how the response of the model is affected by changes in topography, boundary or initial conditions, or in any physical parameter such as the friction coefficient [6].

The sensitivity of a model output (called variable hereafter) to a given parameter is usually defined as the partial derivative of this variable with respect to the parameter. This derivative can be computed using either a discrete or a continuous approach. In the discrete approach, the governing equations for the model variables are first solved numerically and the results are then differentiated with respect to the parameter of interest. Typically, the classical empirical (or finite difference) approach consists in performing two simulations with two slightly different values of the parameter of interest and computing the difference between both results, normalized by the parameter variation. An example of another efficient approach, known as complex differentiation, can be found in [13]. These methods are best-suited to the analysis of complex models dealing with complex geometries, or to the analysis of model response, where the transfer function between the model inputs and outputs is not known exactly. Continuous approaches can be used when the governing equations for the model are known. Differentiating these equations with respect to the parameter of interest leads to the sensitivity equations, that can be solved analytically or numerically.

Both the discrete and continuous approaches, however, use the assumption that the solution of the governing equations is continuous and differentiable with respect to the parameter. They meet problems when the model output becomes discontinuous [9]. This is the case, for example, of the solutions of the shallow water equations in the presence of hydraulic jumps or moving bores. The non-differentiable character of the flow solution yields locally infinite sensitivity values across discontinuities. A sensitivity source term, in the form of a Dirac function, can be introduced at shocks locations to avoid this kind of behaviour [1]. This approach was acknowledged for scalar hyperbolic problems using a different reasoning in [8] and applied to design numerical methods for hyperbolic systems of conservation laws [4, 7]. In these papers however, the solutions proposed for the accurate discretization of the Dirac source term in the framework of shock-capturing numerical methods prove not to be entirely satisfactory in a number of cases [7].

The purpose of this paper is to present a finite volume-based numerical technique for the sensitivity analysis of the one-dimensional Shallow Water Equations (SWEs) with scalar transport in the continuous and discontinuous cases. The difficulties encountered in the previous papers for the treatment of the Dirac source term have been overcome here. 
The SWEs and the derivation of the Shallow Water Sensitivity Equations (SWSEs) are presented in Section 2 and the expressions of the sensitivity source term, initial and boundary conditions are derived. Section 3 is devoted to the detailed presentation of the numerical technique for discontinuous solutions, with a focus on source term and boundary condition discretization. Application examples are provided in Section 4 concerning the sensitivity analysis to the Manning's friction coefficient, and to initial and boundary conditions in a rectangular channel. An application to a trapezoidal channel is also provided. Section 5 is devoted to concluding remarks.

\section{Governing equations}

\subsection{The sensitivity equations}

The development of the sensitivity equations is illustrated using the one-dimensional shallow water equations with passive scalar transport. These equations form a $3 \times 3$ hyperbolic system of conservation laws (HSCL), where the first two equations account for mass and momentum conservation and the third one is a scalar transport equation. They can be written in vector form as

$$
\begin{gathered}
\frac{\partial \mathbf{U}}{\partial t}+\frac{\partial \mathbf{F}}{\partial x}=\mathbf{S} \\
\mathbf{U}(x, 0)=\mathbf{U}_{0}(x) \\
\mathbf{U}\left(x_{b}, t\right)=\mathbf{U}_{b}(t)
\end{gathered}
$$

where $\mathbf{U}$ is the vector of the conserved variables, $\mathbf{F}$ the flux vector and $\mathbf{S}$ the source term; the subscript $b$ denotes the domain boundary and the subscript 0 the initial condition. $\mathbf{U}, \mathbf{F}$, and $\mathbf{S}$ are defined as

$$
\mathbf{U}=\left(\begin{array}{c}
h \\
h u \\
h v
\end{array}\right)=\left(\begin{array}{c}
h \\
q \\
r
\end{array}\right), \mathbf{F}=\left(\begin{array}{c}
q \\
q^{2} / h+g h^{2} / 2 \\
q v
\end{array}\right), \mathbf{S}=\left(\begin{array}{c}
0 \\
g h\left(S_{0}-S_{f}\right) \\
0
\end{array}\right)
$$

where $g$ is the gravitational acceleration, $h$ the water depth, $u$ the flow velocity ( $q=h u$ the unit discharge), $S_{0}=-\partial z_{b} / \partial x$ the bottom slope (with $z_{b}$ the bottom elevation), $S_{f}$ the friction slope (defined in subsection 2.3 ) and $v$ can be any variable subjected to passive scalar transport (for example, $v$ can be the concentration of a pollutant in $\mathrm{kg} \cdot \mathrm{s}^{-1}$ and $r=h v$, the conserved quantity of pollutant in the flow).

The flux function $\mathbf{F}$ and the source term $\mathbf{S}$ defined in Eq. (2) depend on the flow solution $\mathbf{U}$ and on several parameters, such as the gravitational acceleration, the friction coefficient $n_{M}$, the initial and/or boundary conditions, etc. The notation $\mathbf{F}(\mathbf{U}, \psi)$ and $\mathbf{S}(\mathbf{U}, \psi)$ is used in the following, where $\psi$ denotes any of the parameters on which $\mathbf{F}$ and $\mathbf{S}$ may depend. A sensitivity analysis with respect to a given parameter $\psi$ consists in studying the influence of a small variation in this parameter on the solution of the flow equations. The governing equations for the sensitivity (called sensitivity equations hereafter) are obtained by differentiating the flow equation (1a) with respect to the parameter $\psi$.

The parameter $\psi$ is perturbed by a function $\tilde{\psi}(x, t)$ that is non zero only on a sub-domain of the problem domain and defined as

$$
\tilde{\psi}(x, t)=\psi_{0} \varepsilon(x, t)
$$

where $\varepsilon(x, t)$ is the so-called perturbation indicator, with $\varepsilon=0$ in the regions where the parameter remains unchanged and $\varepsilon=1$ in the regions where $\psi$ is modified for the purpose of the sensitivity analysis. Perturbing $\psi$ yields a perturbation $\tilde{\mathbf{U}}$ in the solution $\mathbf{U}$ of $(1 \mathrm{a})$ :

$$
\frac{\partial(\mathbf{U}+\tilde{\mathbf{U}})}{\partial t}+\frac{\partial \mathbf{F}(\mathbf{U}+\tilde{\mathbf{U}}, \psi+\tilde{\psi})}{\partial x}=\mathbf{S}(\mathbf{U}+\tilde{\mathbf{U}}, \psi+\tilde{\psi})
$$

The sensitivity $\mathbf{s}$ of $\mathbf{U}$ to $\psi$ is defined as the partial derivative of $\mathbf{U}$ with respect to $\psi$

$$
\mathbf{s} \equiv \frac{\partial \mathbf{U}}{\partial \psi}=\lim _{\psi_{0} \rightarrow 0} \frac{\tilde{\mathbf{U}}}{\psi_{0}}
$$

Substracting Eq. (1a) from (4) and using a first-order Taylor series expansion in the limit of small $\tilde{\psi}$ and $\tilde{\mathbf{U}}$ yields

$$
\frac{\partial \tilde{\mathbf{U}}}{\partial t}+\frac{\partial}{\partial x}\left(\frac{\partial \mathbf{F}}{\partial \psi} \tilde{\psi}+\frac{\partial \mathbf{F}}{\partial \mathbf{U}} \tilde{\mathbf{U}}\right)=\frac{\partial \mathbf{S}}{\partial \psi} \tilde{\psi}+\frac{\partial \mathbf{S}}{\partial \mathbf{U}} \tilde{\mathbf{U}}
$$


Substituting Eqs. (3) and (5) into (6) and simplifying by $\psi_{0}$ leads to the following PDE in $\mathbf{s}$

$$
\frac{\partial \mathbf{s}}{\partial t}+\frac{\partial}{\partial x}\left(\frac{\partial \mathbf{F}}{\partial \mathbf{U}} \mathbf{s}\right)=\frac{\partial \mathbf{S}}{\partial \mathbf{U}} \mathbf{s}+\frac{\partial \mathbf{S}}{\partial \psi} \varepsilon-\frac{\partial}{\partial x}\left(\frac{\partial \mathbf{F}}{\partial \psi} \varepsilon\right)
$$

Eq. (7) is rewritten as

$$
\begin{gathered}
\frac{\partial \mathbf{s}}{\partial t}+\frac{\partial \mathbf{G}}{\partial x}=\mathbf{Q} \\
\mathbf{s}=\left(\begin{array}{c}
\eta \\
\theta \\
\rho
\end{array}\right) \equiv\left(\begin{array}{c}
\partial h / \partial \psi \\
\partial q / \partial \psi \\
\partial r / \partial \psi
\end{array}\right) \\
\mathbf{G}=\frac{\partial \mathbf{F}}{\partial \mathbf{U}} \mathbf{s}=\left(\begin{array}{ccc}
0 & 1 & 0 \\
c^{2}-u^{2} & 2 u & 0 \\
-u v & v & u
\end{array}\right) \mathbf{s}=\left(\begin{array}{c}
\theta \\
\left(c^{2}-u^{2}\right) \eta+2 u \theta \\
-u v \eta+\theta v+u \rho
\end{array}\right) \\
\mathbf{Q}=\frac{\partial \mathbf{S}}{\partial \mathbf{U}} \mathbf{s}+\frac{\partial \mathbf{S}}{\partial \psi} \varepsilon-\frac{\partial}{\partial x}\left(\frac{\partial \mathbf{F}}{\partial \psi} \varepsilon\right)
\end{gathered}
$$

where $c=(g h)^{1 / 2}$ is the propagation speed of the waves in the fluid at rest. Eqs. (8) are the conservation form of the Shallow Water Sensitivity Equations (SWSEs). The source term $\mathbf{Q}$ defined in Eq. (8d) is the only one that depends on the nature of the parameter $\psi$; its expression is given in subsections 2.3 to 2.5 (after the treatment of discontinuous solutions).

\subsection{Sensitivity equations for discontinuous flow problems}

The sensitivity partial derivative equation (8a) is derived using the assumption that the flow solution $\mathbf{U}$ is continuous and differentiable with respect to the parameter $\psi$ over the solution domain. Discontinuous solutions (e.g. shocks such as hydraulic jumps, moving bores, etc.) are not differentiable with respect to the parameter $\psi$ in the general case and the governing equations must be modified so as to account for extra terms in the balance equations (see $[1,4,8,7]$ ). Indeed, consider a shock moving at a speed $c_{s}$ with leftand right-hand values $\mathbf{U}_{\mathrm{L}}$ and $\mathbf{U}_{\mathrm{R}}$ for the flow variable $\mathbf{U}$ and left- and right-hand values $\mathbf{s}_{\mathrm{L}}$ and $\mathbf{s}_{\mathrm{R}}$ for the sensitivity s; applying the so-called Rankine-Hugoniot conditions (or jump relationship) to a control volume that contains the shock leads to the following equality for the flow variables

$$
\left(\mathbf{U}_{\mathrm{L}}-\mathbf{U}_{\mathrm{R}}\right) c_{s}=\mathbf{F}_{\mathrm{L}}-\mathbf{F}_{\mathrm{R}}
$$

The jump relationship for the sensitivity variables is more complex. As shown in [1] and generalized in $[8,7]$ for a non-uniform perturbation indicator $\varepsilon$, the perturbation $\tilde{\psi}$ yields variations $\tilde{\mathbf{U}}_{\mathrm{L}}$ and $\tilde{\mathbf{U}}_{\mathrm{R}}$ in the values of $\mathbf{U}_{\mathrm{L}}$ and $\mathbf{U}_{\mathrm{R}}$ respectively. This also causes a variation $\tilde{c}_{s}$ in the speed $c_{s}$ of the shock, and consequently, the shock abscissa $x_{s}$ is subject to a change $\tilde{x}_{s}$. The perturbed values of $\mathbf{U}$ and $\mathbf{F}$ on the left- and right-hand side of the shock are then given by

$$
\begin{aligned}
\mathbf{U}_{\mathrm{L}}^{(P)} & =\mathbf{U}_{\mathrm{L}}+\tilde{\mathbf{U}}_{\mathrm{L}}+\tilde{x}_{s} \frac{\partial \mathbf{U}_{\mathrm{L}}}{\partial x} \\
\mathbf{U}_{\mathrm{R}}^{(P)} & =\mathbf{U}_{\mathrm{R}}+\tilde{\mathbf{U}}_{\mathrm{R}}+\tilde{x}_{s} \frac{\partial \mathbf{U}_{\mathrm{R}}}{\partial x} \\
\mathbf{F}_{\mathrm{L}}^{(P)} & =\mathbf{F}_{\mathrm{L}}+\tilde{\mathbf{F}}_{\mathrm{L}}+\tilde{x}_{s} \frac{\partial \mathbf{F}_{\mathrm{L}}}{\partial x} \\
\mathbf{F}_{\mathrm{R}}^{(P)} & =\mathbf{F}_{\mathrm{R}}+\tilde{\mathbf{F}}_{\mathrm{R}}+\tilde{x}_{s} \frac{\partial \mathbf{F}_{\mathrm{R}}}{\partial x}
\end{aligned}
$$

where the superscripts $(P)$ and $(x)$ respectively denote the perturbed value and the derivative with respect to space of the variable of concern. The jump relationship (9) then becomes

$$
\left(\mathbf{U}_{\mathrm{L}}^{(P)}-\mathbf{U}_{\mathrm{R}}^{(P)}\right)\left(c_{s}+\tilde{c}_{s}\right)=\mathbf{F}_{\mathrm{L}}^{(P)}-\mathbf{F}_{\mathrm{R}}^{(P)}
$$

Substituting (10) into (11) yields

$$
\left[\mathbf{U}_{\mathrm{L}}+\tilde{\mathbf{U}}_{\mathrm{L}}+\tilde{x}_{s} \frac{\partial \mathbf{U}_{\mathrm{L}}}{\partial x}-\left(\mathbf{U}_{\mathrm{R}}+\tilde{\mathbf{U}}_{\mathrm{R}}+\tilde{x}_{s} \frac{\partial \mathbf{U}_{\mathrm{R}}}{\partial x}\right)\right]\left(c_{s}+\tilde{c}_{s}\right)=\mathbf{F}_{\mathrm{L}}+\tilde{\mathbf{F}}_{\mathrm{L}}+\tilde{x}_{s} \frac{\partial \mathbf{F}_{\mathrm{L}}}{\partial x}-\left(\mathbf{F}_{\mathrm{R}}+\tilde{\mathbf{F}}_{\mathrm{R}}+\tilde{x}_{s} \frac{\partial \mathbf{F}_{\mathrm{R}}}{\partial x}\right)
$$


Subtracting (9) from (12) and retaining only the first-order terms (in the limit of small $\tilde{c_{s}}, \tilde{x_{s}}$ and $\tilde{\mathbf{U}}$ ) yields

$$
\left(\mathbf{U}_{\mathrm{L}}-\mathbf{U}_{\mathrm{R}}\right) \tilde{c}_{s}+\left(\tilde{\mathbf{U}}_{\mathrm{L}}-\tilde{\mathbf{U}}_{\mathrm{R}}\right) c_{s}+\tilde{x}_{s} \frac{\partial}{\partial x}\left(\mathbf{U}_{\mathrm{L}}-\mathbf{U}_{\mathrm{R}}\right) c_{s}=\tilde{\mathbf{F}}_{\mathrm{L}}-\tilde{\mathbf{F}}_{\mathrm{R}}+\tilde{x}_{s} \frac{\partial}{\partial x}\left(\mathbf{F}_{\mathrm{L}}-\mathbf{F}_{\mathrm{R}}\right)
$$

Noticing that

$$
\begin{gathered}
\tilde{\mathbf{U}}=\psi_{0} \mathbf{s} \\
\tilde{\mathbf{F}}=\psi_{0} \mathbf{G}+(\partial \mathbf{F} / \partial \psi) \varepsilon \psi_{0} \\
\tilde{x}_{s}=\left(\partial x_{s} / \partial \psi\right) \psi_{0} \\
\tilde{c}_{s}=\left(\partial c_{s} / \partial \psi\right) \psi_{0}
\end{gathered}
$$

and simplifying by $\psi_{0}$, the complete jump relationship for the sensitivity can be written as

$$
\begin{aligned}
\left(\mathbf{s}_{\mathrm{L}}-\mathbf{s}_{\mathrm{R}}\right) c_{s}=\mathbf{G}_{\mathrm{L}}-\mathbf{G}_{\mathrm{R}}-\frac{\partial c_{s}}{\partial \psi}\left(\mathbf{U}_{\mathrm{L}}-\mathbf{U}_{\mathrm{R}}\right) & -\frac{\partial x_{s}}{\partial \psi}\left[\left(\frac{\partial \mathbf{U}}{\partial x}\right)_{\mathrm{L}}-\left(\frac{\partial \mathbf{U}}{\partial x}\right)_{\mathrm{R}}\right] c_{s} \\
& +\left(\frac{\partial \mathbf{F}}{\partial \psi}\right)_{\mathrm{L}}-\left(\frac{\partial \mathbf{F}}{\partial \psi}\right)_{\mathrm{R}}+\frac{\partial x_{s}}{\partial \psi}\left[\left(\frac{\partial \mathbf{F}}{\partial x}\right)_{\mathrm{L}}-\left(\frac{\partial \mathbf{F}}{\partial x}\right)_{\mathrm{R}}\right]
\end{aligned}
$$

The following notation is used for the additional term in (15)

$$
\begin{aligned}
& \mathbf{R}=-\frac{\partial c_{s}}{\partial \psi}\left(\mathbf{U}_{\mathrm{L}}-\mathbf{U}_{\mathrm{R}}\right)-\frac{\partial x_{s}}{\partial \psi}\left[\left(\frac{\partial \mathbf{U}}{\partial x}\right)_{\mathrm{L}}-\left(\frac{\partial \mathbf{U}}{\partial x}\right)_{\mathrm{R}}\right] c_{s} \\
& +\left(\frac{\partial \mathbf{F}}{\partial \psi} \varepsilon\right)_{\mathrm{L}}-\left(\frac{\partial \mathbf{F}}{\partial \psi} \varepsilon\right)_{\mathrm{R}}+\frac{\partial x_{s}}{\partial \psi}\left[\left(\frac{\partial \mathbf{F}}{\partial x}\right)_{\mathrm{L}}-\left(\frac{\partial \mathbf{F}}{\partial x}\right)_{\mathrm{R}}\right]
\end{aligned}
$$

Note that this rather complex expression can be simplified in the case of the solution of the Riemann problem (see section 3.2). This source term being non-zero only at points where $\mathbf{U}$ is discontinuous, Eq. (8a) can be rewritten for continuous and discontinuous solutions

$$
\frac{\partial \mathbf{s}}{\partial t}+\frac{\partial \mathbf{G}}{\partial x}=\mathbf{Q}+\mathbf{R} \delta_{s}
$$

where $\delta_{s}$ denotes the Dirac distribution located at the shock. The only term in (17) that depends on the nature of the parameter $\psi$ is the source term $\mathbf{Q}$ defined by Eq. (8d). In the following subsections, the expression of $\mathbf{Q}$ is provided for the sensitivity to the friction coefficient and to initial and boundaries conditions.

\subsection{Sensitivity to the friction coefficient}

In what follows, the friction slope is assumed to obey a classical Manning-Strickler law under the wide channel approximation

$$
S_{f}=q|q| n_{M}^{2} h^{-10 / 3}
$$

where $n_{M}$ is Manning's friction coefficient. The expression of $\mathbf{Q}$ can be derived for the particular case where the parameter $\psi=n_{M}$. Since $\mathbf{F}$ is not a function of $n_{M}, \partial \mathbf{F} / \partial \psi=0$ and

$$
\mathbf{Q}=\frac{\partial \mathbf{S}}{\partial \mathbf{U}} \mathbf{s}+\frac{\partial \mathbf{S}}{\partial \psi} \varepsilon_{n_{M}}
$$

with

$$
\frac{\partial \mathbf{S}}{\partial \mathbf{U}} \mathbf{S}=\left(\begin{array}{c}
0 \\
g\left(S_{0}+7 / 3 S_{f}\right) \eta-2 g n_{M}^{2}|q| h^{-7 / 3} \theta \\
0
\end{array}\right)
$$

and

$$
\frac{\partial \mathbf{S}}{\partial \psi} \varepsilon_{n_{M}}=\left(\begin{array}{c}
0 \\
-2 g h \frac{S_{f}}{n_{M}} \varepsilon_{n_{M}} \\
0
\end{array}\right)
$$

where $\varepsilon_{n_{M}}=0$ in regions where the friction coefficient is unchanged and $\varepsilon_{n_{M}}=1$ in the regions where it is perturbed for the sensitivity analysis. 


\subsection{Sensitivity to initial conditions}

The sensitivity to the initial conditions is studied by defining $\psi$ as one of the flow variables $(h, q$, or $r)$ at $t=0$. Considering the sensitivity to the initial conditions yields

$$
\varepsilon(x, t)=0 \quad \forall t>0
$$

The expression of $\mathbf{Q}$ then reduces to the term $(\partial \mathbf{S} / \partial \mathbf{U}) \mathbf{s}$ defined by $(20)$. The initial condition for the SWSEs (8) is given by

$$
\mathbf{s}(x, 0)=\left(\begin{array}{c}
\varepsilon_{h} \\
\varepsilon_{q} \\
\varepsilon_{r}
\end{array}\right)
$$

where $\varepsilon_{h}, \varepsilon_{q}$ and $\varepsilon_{r}$ are the perturbation indicators respectively for $h(x, 0), q(x, 0)$ and $r(x, 0)$. Assume for instance that a dam-break problem [17] is to be solved for the following initial conditions

$$
\begin{gathered}
h(x, 0)= \begin{cases}h_{\mathrm{L}} & \text { for } x<x_{0} \\
h_{\mathrm{R}} & \text { for } x \geq x_{0}\end{cases} \\
q(x, 0)=0 \quad \forall x \\
r(x, 0)=0 \quad \forall x
\end{gathered}
$$

where $x_{0}$ is the abscissa of the dam. Assume that the purpose of the analysis is to study the sensitivity of the flow solution to the water depth $h_{\mathrm{L}}$ on the left-hand side of the dam. Then the perturbation indicators in (23) must be defined as

$$
\begin{gathered}
\varepsilon_{h}(x, 0)= \begin{cases}1 & \text { for } x<x_{0} \\
0 & \text { for } x \geq x_{0}\end{cases} \\
\varepsilon_{q}(x, 0)=0 \quad \forall x \\
\varepsilon_{r}(x, 0)=0 \quad \forall x
\end{gathered}
$$

\subsection{Boundary conditions}

Boundary conditions for the flow are relationships in the form

$$
f_{i}(\mathbf{U}, \psi, t)=0
$$

As shown in [2], as many boundary conditions $f_{i}$ must be supplied at a given boundary as there are characteristics entering the domain. It stems from Eq. (8c) that the Jacobian matrix $\partial \mathbf{G} / \partial \mathbf{s}$ for the sensitivity is identical to the Jacobian matrix $\partial \mathbf{F} / \partial \mathbf{U}$ for the original flow problem. Consequently, the characteristics for the sensitivity problem are identical to the characteristics for the flow problem and the sensitivity problem requires as many boundary conditions as the original flow problem. These conditions are obtained by differentiating Eq. (26) with respect to $\psi$

$$
\frac{\mathrm{d} f_{i}}{\mathrm{~d} \psi}=\frac{\partial f_{i}}{\partial h} \eta+\frac{\partial f_{i}}{\partial q} \theta+\frac{\partial f_{i}}{\partial r} \rho
$$

Two main situations may occur:

1. The sensitivity analysis is carried out with respect to the initial conditions or to a hydrodynamic parameter. In this case, the boundary conditions are not influenced by the value of $\psi$ and Eq. (27) becomes

$$
\frac{\partial f_{i}}{\partial h} \eta+\frac{\partial f_{i}}{\partial q} \theta+\frac{\partial f_{i}}{\partial r} \rho=0
$$


2. The sensitivity analysis is carried out with respect to the boundary condition. This is the case for instance, when the numerical value of the boundary condition to be prescribed is not known with certainty, or measured with a certain imprecision. For example, assuming that a known water depth $h_{b}$ is to be prescribed at a boundary, (26) becomes

$$
f=h-h_{b}=0
$$

Applying (27) with $\psi=h_{b}$, yields the following sensitivity boundary condition

$$
\eta=1
$$

For a prescribed unit discharge $q_{b}, f=q-q_{b}=0$ and the sensitivity boundary condition is

$$
\theta=1
$$

and for a prescribed quantity of the transported variable, $f=r-r_{b}=0$ and

$$
\rho=1
$$

\section{Numerical technique}

This section deals with the numerical technique developed to solve the SWSEs. The finite volume discretization is briefly recalled in section 3.1, then the fluxes calculation using the Riemann problem is detailed in section 3.2. Section 3.3 is devoted to source terms calculation in the continuous case and section 3.4 to shock detection and to the calculation of the specific source term $\mathbf{R}$. Section 3.5 describes the discretization of boundary conditions.

\subsection{Finite volume discretization}

The flow and sensitivity equations (Eqs. (1a) and (17)) are discretized using a finite volume formulation:

$$
\begin{gathered}
\mathbf{U}_{i}^{n+1}=\mathbf{U}_{i}^{n}-\frac{\Delta t}{\Delta x_{i}}\left(\mathbf{F}_{i-1 / 2}^{n+1 / 2}-\mathbf{F}_{i+1 / 2}^{n+1 / 2}\right)+\Delta t \mathbf{S}_{i}^{n+1 / 2} \\
\mathbf{s}_{i}^{n+1}=\mathbf{s}_{i}^{n}-\frac{\Delta t}{\Delta x_{i}}\left(\mathbf{G}_{i-1 / 2}^{n+1 / 2}-\mathbf{G}_{i+1 / 2}^{n+1 / 2}+\mathbf{R}_{i-1 / 2}^{n+1 / 2}+\mathbf{R}_{i+1 / 2}^{n+1 / 2}\right)+\Delta t \mathbf{Q}_{i}^{n+1 / 2}
\end{gathered}
$$

where $\Delta t$ is the computational time step, $\Delta x_{i}$ is the width of the cell $i, \mathbf{U}_{i}^{n}$ and $\mathbf{s}_{i}^{n}$ are respectively the average value of $\mathbf{U}$ and $\mathbf{s}$ over the cell $i$ at the time level $n, \mathbf{F}_{i-1 / 2}^{n+1 / 2}$ and $\mathbf{G}_{i-1 / 2}^{n+1 / 2}$ are the average values of the fluxes $\mathbf{F}$ and $\mathbf{G}$ through the interface $i-1 / 2$ (between the cells $i-1$ and $i$ ) between the time levels $n$ and $n+1, \mathbf{S}_{i}^{n+1 / 2}$ and $\mathbf{Q}_{i}^{n+1 / 2}$ are the average values of $\mathbf{S}$ and $\mathbf{Q}$ over the cell $i$ between the time levels $n$ and $n+1, \mathbf{R}_{i-1 / 2}^{n+1 / 2}\left(\operatorname{resp} \mathbf{R}_{i+1 / 2}^{n+1 / 2}\right)$ is the contribution of the Dirac source terms possibly generated by shocks propagating from the cell $i-1$ into the cell $i$ (resp. $i$ into $i+1$ ) between time levels $n$ and $n+1$.

\subsection{Fluxes calculation}

The fluxes $\mathbf{F}$ and $\mathbf{G}$ in Eqs (33) can be computed by solving Riemann problems. However, the Riemann problem for the sensitivity cannot be considered independently from the one for the flow variable, because the Jacobian matrix $\partial \mathbf{F} / \partial \mathbf{U}$ (used in the definition of $\mathbf{G}$, Eq. (8c)) depends on the flow solution $\mathbf{U}$. A single Riemann problem is thus defined for the hyperbolic part of the governing equations (1a) and (8a) at a given interface $i+1 / 2$ (between cells $i$ and $i+1$ )

$$
\begin{gathered}
\frac{\partial \mathbf{U}}{\partial t}+\frac{\partial \mathbf{F}}{\partial x}=0 \\
\frac{\partial \mathbf{s}}{\partial t}+\frac{\partial \mathbf{G}}{\partial x}=\mathbf{R} \delta_{s} \\
(\mathbf{U}, \mathbf{s})(x, 0)= \begin{cases}\left(\mathbf{U}_{\mathrm{L}}, \mathbf{s}_{\mathrm{L}}\right) & \text { for } x<x_{i+1 / 2} \\
\left(\mathbf{U}_{\mathrm{R}}, \mathbf{s}_{\mathrm{R}}\right) & \text { for } x \geq x_{i+1 / 2}\end{cases}
\end{gathered}
$$

where $x_{i+1 / 2}$ is the abscissa of the interface. These equations stem from (1) and (17) where $\mathbf{U}$ and $\mathbf{F}$ are constant on both sides of the discontinuity and where no source term is considered (i.e. $\mathbf{S}$ and $\mathbf{Q}$ are nil). 
In this case, the $x$-derivatives vanish in the expression of $\mathbf{R}$, Eq. (16), as well as the terms in $\frac{\partial \mathbf{F}}{\partial \psi} \varepsilon$ (as a consequence of $\mathbf{Q}=0$ in Eq. (8d)) leading to the following simplification

$$
\mathbf{R}=-\frac{\partial c_{s}}{\partial \psi}\left(\mathbf{U}_{\mathrm{L}}-\mathbf{U}_{\mathrm{R}}\right)
$$

The two corresponding $3 \times 3$ HSCLs defined by equations (1a) and (17) have the same eigenvalues. These three eigenvalues are real and distinct

$$
\left(\begin{array}{l}
\lambda^{(1)} \\
\lambda^{(2)} \\
\lambda^{(3)}
\end{array}\right)=\left(\begin{array}{c}
u-c \\
u \\
u+c
\end{array}\right)
$$

The general solution of the Riemann problem for the flow solution is known to be made of three waves separating two internal regions of constant state from the left- and right states of the Riemann problem. The HLL/HLLC Riemann solver $[10,18]$ is based on the a priori assumption that the first and third waves $\left(\mathrm{d} x / \mathrm{d} t=\lambda^{(1)}\right.$ and $\left.\mathrm{d} x / \mathrm{d} t=\lambda^{(3)}\right)$ are discontinuities. The exact nature of such discontinuities (shocks or contact discontinuities), as well as whether such waves satisfy the entropy condition, does not need to be known in the solution process. The second wave $\left(\mathrm{d} x / \mathrm{d} t=\lambda^{(2)}\right)$ is a contact wave. The fluxes are computed using the original HLLC Riemann solver for the flow problem and a modified HLLC solver for the sensitivity problem.

Using an a priori approximation $\lambda^{-}\left(\operatorname{resp} . \lambda^{+}\right)$of $\lambda^{(1)}\left(\operatorname{resp} . \lambda^{(3)}\right)$, allows the Rankine-Hugoniot conditions to be written, for the first two components of $(1 \mathrm{a})$, as

$$
\begin{aligned}
& \left(\mathbf{U}_{\mathrm{L}}-\mathbf{U}_{*}\right) \lambda^{-}=\mathbf{F}_{\mathrm{L}}-\mathbf{F}_{*} \\
& \left(\mathbf{U}_{\mathrm{R}}-\mathbf{U}_{*}\right) \lambda^{+}=\mathbf{F}_{\mathrm{R}}-\mathbf{F}_{*}
\end{aligned}
$$

where the subscript $*$ indicates the value of the variable $\mathbf{U}$ and the flux $\mathbf{F}$ in the intermediate region of constant state. Solving Eqs. (37) for $\mathbf{U}_{*}$ and $\mathbf{F}_{*}$ yields

$$
\begin{gathered}
\mathbf{U}_{*}=\frac{-\lambda^{-} \mathbf{U}_{\mathrm{L}}+\lambda^{+} \mathbf{U}_{\mathrm{R}}+\mathbf{F}_{\mathrm{L}}-\mathbf{F}_{\mathrm{R}}}{\lambda^{+}-\lambda^{-}} \\
\mathbf{F}_{*}=\frac{\lambda^{+} \mathbf{F}_{\mathrm{L}}-\lambda^{-} \mathbf{F}_{\mathrm{R}}-\lambda^{-} \lambda^{+}\left(\mathbf{U}_{\mathrm{L}}-\mathbf{U}_{\mathrm{R}}\right)}{\lambda^{+}-\lambda^{-}}
\end{gathered}
$$

Depending on the values of $\lambda^{(1)}$ and $\lambda^{(3)}$, the interface $i+1 / 2$ may not be located within the intermediate region of constant state, but in the left or right state. The following estimates for $\lambda^{(1)}$ and $\lambda^{(3)}$ allow all possible flow situations (subsonic/sub-critical and supersonic/supercritical) to be handled by a single formula $[3]$

$$
\begin{aligned}
& \lambda^{-}=\min \left(u_{\mathrm{L}}-c_{\mathrm{L}}, u_{\mathrm{R}}-c_{\mathrm{R}}, 0\right) \\
& \lambda^{+}=\max \left(u_{\mathrm{L}}+c_{\mathrm{L}}, u_{\mathrm{R}}+c_{\mathrm{R}}, 0\right)
\end{aligned}
$$

The first and second components of the flux $\mathbf{F}=\left(\mathbf{F}_{\mathbf{1}}, \mathbf{F}_{\mathbf{2}}, \mathbf{F}_{\mathbf{3}}\right)$ can be estimated using Eq. (39) between the cells $i$ and $i+1$

$$
\begin{gathered}
\left(\mathbf{F}_{\mathbf{1}}\right)_{i+1 / 2}^{n+1 / 2}=\frac{\lambda^{+} q_{\mathrm{L}}-\lambda^{-} q_{\mathrm{R}}-\lambda^{-} \lambda^{+}\left(z_{\mathrm{L}}-z_{\mathrm{R}}\right)}{\lambda^{+}-\lambda^{-}} \\
\left(\mathbf{F}_{\mathbf{2}}\right)_{i+1 / 2}^{n+1 / 2}=\frac{\lambda^{+} \mathbf{F}_{2, \mathrm{~L}}-\lambda^{-} \mathbf{F}_{2, \mathrm{R}}-\lambda^{-} \lambda^{+}\left(q_{\mathrm{L}}-q_{\mathrm{R}}\right)}{\lambda^{+}-\lambda^{-}}
\end{gathered}
$$

Note that in the first component of (39), the free surface elevations $z_{\mathrm{L}}$ and $z_{\mathrm{R}}$ are used instead of the original conserved variables $h_{\mathrm{L}}$ and $h_{\mathrm{R}}$ for well-balancing of the flux and topographical source term [14].

From numerical experiments, it has been shown in [5] that applying this procedure to the third component of the flow HSCL (1a) may cause unphysical oscillations, sometimes leading to instability in the solution of the scalar transport equation. An alternative formulation must be used for the contact discontinuity (note that this was also the case in the HLLC Riemann solver [18]). The third component is thus estimated using the following expression

$$
\left(\mathbf{F}_{\mathbf{3}}\right)_{i+1 / 2}^{n+1 / 2}= \begin{cases}\left(\mathbf{F}_{\mathbf{1}}\right)_{i+1 / 2}^{n+1 / 2} v_{\mathrm{L}} & \text { if } \mathrm{u} \geq 0 \\ \left(\mathbf{F}_{\mathbf{1}}\right)_{i+1 / 2}^{n+1 / 2} v_{\mathrm{R}} & \text { if } \mathrm{u}<0\end{cases}
$$

taking into account the characteristic equation in the passive variable $v$

$$
\frac{\mathrm{d} v}{\mathrm{~d} t}=0 \text { for } \frac{\mathrm{d} x}{\mathrm{~d} t}=u
$$


The two first components of the sensitivity flux $\mathbf{G}_{i+1 / 2}^{n+1 / 2}$ are obtained by extending Eqs. (41) to the sensitivity balance

$$
\begin{gathered}
\left(\mathbf{G}_{\mathbf{1}}\right)_{i+1 / 2}^{n+1 / 2}=\frac{\lambda^{+} \theta_{\mathrm{L}}-\lambda^{-} \theta_{\mathrm{R}}-\lambda^{-} \lambda^{+}\left(\eta_{\mathrm{L}}-\eta_{\mathrm{R}}\right)}{\lambda^{+}-\lambda^{-}} \\
\left(\mathbf{G}_{\mathbf{2}}\right)_{i+1 / 2}^{n+1 / 2}=\frac{\lambda^{+} \mathbf{G}_{2, \mathrm{~L}}-\lambda^{-} \mathbf{G}_{2, \mathrm{R}}-\lambda^{-} \lambda^{+}\left(\theta_{\mathrm{L}}-\theta_{\mathrm{R}}\right)}{\lambda^{+}-\lambda^{-}}
\end{gathered}
$$

As mentioned in previous subsections, such a transposition is not valid strictly speaking because of the extra source term $\mathbf{R}$ that may appear at the discontinuities $\lambda^{-}$and $\lambda^{+}$. However, it should be kept in mind that the present section deals with the conservation part of the equations only and that the source term $\mathbf{R}$ is to be treated separately. Numerical experiments [5] indicate that incorporating $\mathbf{R}$ into (44a) does not bring any noticeable change to the quality of the numerical solution.

The issue identified for the third component of the flow HSCL (1a) holds for the sensitivity HSCL (8a). The sensitivity contact wave should be discretized so as to preserve the invariance property of the sensitivity Riemann invariant along this wave. The third component of the sensitivity flux $\mathbf{G}_{i+1 / 2}^{n+1 / 2}$ is thus obtained by extending the treatment proposed for $\left(\mathbf{F}_{\mathbf{3}}\right)_{i+1 / 2}^{n+1 / 2}$ to the sensitivity [5]. The derivation of Eq. (43) with respect to the parameter $\psi$ leads to the following invariance property

$$
\frac{\mathrm{d} \omega}{\mathrm{d} t}=0 \text { for } \frac{\mathrm{d} x}{\mathrm{~d} t}=u
$$

where $\omega$ is the sensitivity of the passive variable $v$. This equation can also be written in non-conservation form

$$
\frac{\partial \omega}{\partial t}+u \frac{\partial w}{\partial x}=0
$$

Moreover, since $r=h v$

$$
\mathbf{s}_{3}=\rho=h \omega+\eta v
$$

and

$$
\mathbf{G}_{3}=\theta v+q \omega
$$

Substituting these two equations into the third component of (8a) yields

$$
\frac{\partial \eta v}{\partial t}+\frac{\partial h \omega}{\partial t}+\frac{\partial \theta v}{\partial x}+\frac{\partial q \omega}{\partial x}=0
$$

expanding the derivatives of $\eta v$ and $\theta v$, substituting the first component of Eq. (1a) and using (46) yields the following equation

$$
\frac{\partial h \omega}{\partial t}+\frac{\partial q \omega}{\partial x}=0
$$

This equation is discretized in a finite volume framework as

$$
(h \omega)_{i}^{n+1}=(h \omega)_{i}^{n}+\frac{\Delta t}{\Delta x}\left[(q \omega)_{i-1 / 2}^{n+1 / 2}-(q \omega)_{i+1 / 2}^{n+1 / 2}\right]
$$

where $q w$ is computed using the invariance property (46)

$$
(q w)_{i+1 / 2}^{n+1 / 2}= \begin{cases}q_{i+1 / 2}^{n+1 / 2} \omega_{\mathrm{L}} & \text { if } \mathrm{u} \geq 0 \\ q_{i+1 / 2}^{n+1 / 2} \omega_{\mathrm{R}} & \text { if } \mathrm{u}<0\end{cases}
$$

Consequently, the third component of the balance equation (33b) is replaced by

$$
\rho^{n+1}=\rho^{n}+\frac{\Delta t}{\Delta x}\left[(q \omega)_{i-1 / 2}^{n+1 / 2}-(q \omega)_{i+1 / 2}^{n+1 / 2}\right]+(\eta v)_{i}^{n+1}-(\eta v)_{i}^{n}
$$

Note that, since the eigenvalues of the Jacobian matrices $\partial \mathbf{F} / \partial \mathbf{U}$ and $\partial \mathbf{G} / \partial \mathbf{s}$ are identical, the stability constraint is the same for the flow and sensitivity problems. The maximum permissible computational time step $\Delta t_{\max }$ is such that the Courant Friedrich Lax number (CFL) associated to the fastest of the waves $u-c$ and $u+c$ is smaller than unity. The stability constraint is therefore

$$
\Delta t_{\max }=\min _{i} \frac{\Delta x_{i}}{\left|u_{i}^{n}\right|+c_{i}^{n}}
$$




\subsection{Source term calculation in the continuous case}

The source term $\mathbf{S}$ in (33a) is computed as

$$
\mathbf{S}_{i}^{n+1 / 2}=\left[\begin{array}{c}
0 \\
g h\left(S_{0}-S_{f}\right) \\
0
\end{array}\right]_{i}^{n+1 / 2}
$$

and discretized using a classical source term upwinding procedure [19]

$$
\mathbf{S}_{i}^{n+1 / 2}=\left[\begin{array}{c}
0 \\
g h\left(S_{0}-S_{f}\right) \\
0
\end{array}\right]_{i, \mathrm{~L}}^{n+1 / 2}+\left[\begin{array}{c}
0 \\
g h\left(S_{0}-S_{f}\right) \\
0
\end{array}\right]_{i, \mathrm{R}}^{n+1 / 2}
$$

where the subscripts $\mathrm{L}$ and $\mathrm{R}$ denote the respective contributions of the left and right interface of the cell $i$ to the total source term. The contributions of the interface $i+1 / 2$ to the cells $i$ and $i+1$ are given by

$$
\begin{aligned}
{\left[g h\left(S_{0}-S_{f}\right)\right]_{i, \mathrm{~L}}^{n+1 / 2} } & =\frac{-\lambda^{-}}{\lambda^{+}-\lambda^{-}} g h_{i+1 / 2}^{n}\left[-\frac{z_{b_{i}}-z_{b_{i+1}}}{\Delta x_{i}}-n_{M}^{2} h_{i+1 / 2}^{-10 / 3} q_{i+1 / 2}\left|q_{i+1 / 2}\right|\right] \\
{\left[g h\left(S_{0}-S_{f}\right)\right]_{i+1, \mathrm{R}}^{n+1 / 2} } & =\frac{\lambda^{+}}{\lambda^{+}-\lambda^{-}} g h_{i+1 / 2}^{n}\left[-\frac{z_{b_{i}}-z_{b_{i+1}}}{\Delta x_{i}}-n_{M}^{2} h_{i+1 / 2}^{-10 / 3} q_{i+1 / 2}\left|q_{i+1 / 2}\right|\right]
\end{aligned}
$$

where the subscript $i+1 / 2$ denotes the mean of the variable values in cells $i$ and $i+1$.

The source term $\mathbf{Q}$ for the sensitivity is computed as

$$
\mathbf{Q}_{i}^{n+1 / 2}=\left(\begin{array}{c}
0 \\
S_{0} g \eta+7 / 3 S_{f} g \eta-2 g n_{M}^{2}|q| h^{-7 / 3} \theta-2 g h \frac{S_{f}}{n_{M}} \varepsilon_{n_{M}} \\
0
\end{array}\right)_{i}^{n+1 / 2}
$$

and is discretized using the same procedure as $\mathbf{S}$.

\subsection{Shock detection and calculation of $\mathbf{R}$}

In $[4,8,7]$ the following criteria were identified as satisfactory. A shock is detected for the wave $\lambda^{-}$if the following conditions are both satisfied

$$
\begin{aligned}
& u_{\mathrm{L}}-c_{\mathrm{L}}>u_{*}-c_{*} \\
& u_{\mathrm{L}}+c_{\mathrm{L}}>u_{*}+c_{*}
\end{aligned}
$$

while a shock is detected for the wave $\lambda^{+}$if

$$
\begin{aligned}
& u_{*}-c_{*}>u_{\mathrm{R}}-c_{\mathrm{R}} \\
& u_{*}+c_{*}>u_{\mathrm{R}}+c_{\mathrm{R}}
\end{aligned}
$$

where $u_{*}$ and $c_{*}$ are respectively the flow velocity and the propagation speed of the waves in the fluid at rest in the intermediate region of constant state.

Note that in the original theory of hyperbolic systems of conservation laws [11], Eq. (59a) and (60b) are necessary and sufficient conditions for shock wave detection. However, in [11] the influence of source terms is not considered. Using these equations alone would lead to detecting a shock even when the water is at rest when the bottom slope is nonzero [4, 7], hence the need for the extra conditions (59b) and (60a).

When a shock is detected, the point source term $\mathbf{R}$ is split into two contributions: the contribution $\mathbf{R}^{-}$ of the wave $\lambda^{-}$and the contribution $\mathbf{R}^{+}$of the wave $\lambda^{+}$. Since the wave $\lambda^{-}$separates the left state of the Riemann problem from the intermediate region of constant state and the wave $\lambda^{+}$separates the right state of the Riemann problem from the intermediate region of constant state, applying (16) yields

$$
\begin{aligned}
& \mathbf{R}^{-}=-\frac{\partial \lambda^{-}}{\partial \psi}\left(\mathbf{U}_{\mathrm{L}}-\mathbf{U}_{*}\right) \\
& \mathbf{R}^{+}=-\frac{\partial \lambda^{+}}{\partial \psi}\left(\mathbf{U}_{*}-\mathbf{U}_{\mathrm{R}}\right)
\end{aligned}
$$


The key idea in the present solver [5] consists in using Eq. (38) for the estimate of $\mathbf{U}^{*}$ and noticing that the wave speeds $\lambda^{-}$and $\lambda^{+}$depend on $\mathbf{U}_{\mathrm{L}}$ and $\mathbf{U}_{\mathrm{R}}$ only in the conservation part of the equations. Consequently, (61a) may be rewritten as

$$
\mathbf{R}^{-}=-\left(\frac{\partial \lambda^{-}}{\partial \mathbf{U}_{\mathrm{L}}} \frac{\partial \mathbf{U}_{\mathrm{L}}}{\partial \psi}+\frac{\partial \lambda^{-}}{\partial \mathbf{U}_{\mathrm{R}}} \frac{\partial \mathbf{U}_{\mathrm{R}}}{\partial \psi}\right)\left(\mathbf{U}_{\mathrm{L}}-\mathbf{U}_{*}\right)
$$

Noticing that $\partial \mathbf{U} / \partial \psi=\mathbf{s},(62)$ becomes

$$
\mathbf{R}^{-}=-\left(\frac{\partial \lambda^{-}}{\partial \mathbf{U}_{\mathrm{L}}} \mathbf{s}_{\mathrm{L}}+\frac{\partial \lambda^{-}}{\partial \mathbf{U}_{\mathrm{R}}} \mathbf{s}_{\mathrm{R}}\right)\left(\mathbf{U}_{\mathrm{L}}-\mathbf{U}_{*}\right)
$$

Applying a similar reasoning to the wave $\lambda^{+}$yields

$$
\mathbf{R}^{+}=-\left(\frac{\partial \lambda^{+}}{\partial \mathbf{U}_{\mathrm{L}}} \mathbf{s}_{\mathrm{L}}+\frac{\partial \lambda^{+}}{\partial \mathbf{U}_{\mathrm{R}}} \mathbf{s}_{\mathrm{R}}\right)\left(\mathbf{U}_{*}-\mathbf{U}_{\mathrm{R}}\right)
$$

It is easy to check that the definitions (40) lead to the following expressions for the row matrices $\partial \lambda^{ \pm} / \partial \mathbf{U}$

$$
\begin{aligned}
& \frac{\partial \lambda^{-}}{\partial \mathbf{U}_{\mathrm{L}}}=\left\{\begin{array}{lll}
\frac{1}{h_{\mathrm{L}}}\left[\begin{array}{lll}
-u_{\mathrm{L}}-\frac{c_{\mathrm{L}}}{2} & 1 & 0
\end{array}\right] & \text { if } u_{\mathrm{L}}-c_{\mathrm{L}}<\min \left(u_{\mathrm{R}}-c_{\mathrm{R}} ; 0\right) \\
0 & \text { otherwise }
\end{array}\right. \\
& \frac{\partial \lambda^{-}}{\partial \mathbf{U}_{\mathrm{R}}}= \begin{cases}\frac{1}{h_{\mathrm{R}}}\left[\begin{array}{lll}
-u_{\mathrm{R}}-\frac{c_{\mathrm{R}}}{2} & 1 & 0
\end{array}\right] & \text { if } u_{\mathrm{R}}-c_{\mathrm{R}}<\min \left(u_{\mathrm{L}}-c_{\mathrm{L}} ; 0\right) \\
{\left[\begin{array}{ccc}
0 & 0 & 0
\end{array}\right]} & \text { otherwise }\end{cases} \\
& \frac{\partial \lambda^{+}}{\partial \mathbf{U}_{\mathrm{L}}}=\left\{\begin{array}{lll}
\frac{1}{h_{\mathrm{L}}}\left[\begin{array}{lll}
-u_{\mathrm{L}}+\frac{c_{\mathrm{L}}}{2} & 1 & 0
\end{array}\right] & \text { if } u_{\mathrm{L}}+c_{\mathrm{L}} \geq \max \left(u_{\mathrm{R}}+c_{\mathrm{R}} ; 0\right) \\
{\left[\begin{array}{lll}
0 & 0 & 0
\end{array}\right]} & \text { otherwise }
\end{array}\right.
\end{aligned}
$$

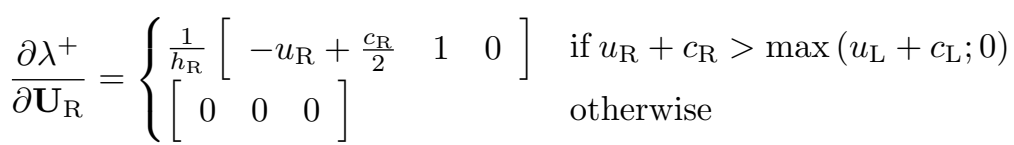

Substituting Eqs (65) into (63) and (64) yields

$$
\begin{aligned}
& \mathbf{R}^{-}= \begin{cases}\frac{1}{h_{\mathrm{L}}}\left[\left(u_{\mathrm{L}}+\frac{c_{\mathrm{L}}}{2}\right) \eta_{\mathrm{L}}-\theta_{\mathrm{L}}\right]\left(\mathbf{U}_{\mathrm{L}}-\mathbf{U}_{*}\right) & \text { if } u_{\mathrm{L}}-c_{\mathrm{L}}<\min \left(u_{\mathrm{R}}-c_{\mathrm{R}} ; 0\right) \\
\frac{1}{h_{\mathrm{R}}}\left[\left(u_{\mathrm{R}}+\frac{c_{\mathrm{R}}}{2}\right) \eta_{\mathrm{R}}-\theta_{\mathrm{R}}\right]\left(\mathbf{U}_{\mathrm{L}}-\mathbf{U}_{*}\right) & \text { if } u_{\mathrm{L}}-c_{\mathrm{L}} \geq \max \left(u_{\mathrm{R}}-c_{\mathrm{R}} ; 0\right)\end{cases} \\
& \mathbf{R}^{+}= \begin{cases}\frac{1}{h_{\mathrm{R}}}\left[\left(u_{\mathrm{R}}-\frac{c_{\mathrm{R}}}{2}\right) \eta_{\mathrm{R}}-\theta_{\mathrm{R}}\right]\left(\mathbf{U}_{*}-\mathbf{U}_{\mathrm{R}}\right) & \text { if } u_{\mathrm{L}}+c_{\mathrm{L}}<\min \left(u_{\mathrm{R}}+c_{\mathrm{R}} ; 0\right) \\
\frac{1}{h_{\mathrm{L}}}\left[\left(u_{\mathrm{L}}-\frac{c_{\mathrm{L}}}{2}\right) \eta_{\mathrm{L}}-\theta_{\mathrm{L}}\right]\left(\mathbf{U}_{*}-\mathbf{U}_{\mathrm{R}}\right) & \text { if } u_{\mathrm{L}}+c_{\mathrm{L}} \geq \max \left(u_{\mathrm{R}}+c_{\mathrm{R}} ; 0\right)\end{cases}
\end{aligned}
$$

\subsection{Discretization of boundary conditions}

\subsubsection{Shallow water equations}

Three types of boundary conditions are considered hereafter for the equations of mass and momentum conservation: prescribed water depth, prescribed discharge and prescribed stage-discharge relationship. For the sake of conciseness, only the left-hand boundary of the domain is considered, the transposition to a righthand boundary being straightforward. Only subcritical conditions are considered hereafter, the supercritical case being straightforward.

1. Prescribed water depth. Assume that the water depth $h_{b}$ is to be prescribed at the left-hand boundary. Then, the water depth and the sensitivity at the left-hand interface $1 / 2$ of the computational cell 1 are given by

$$
\begin{aligned}
& h_{1 / 2}^{n+1 / 2}=h_{b} \\
& \eta_{1 / 2}^{n+1 / 2}=\eta_{b}
\end{aligned}
$$

with $\eta_{b}=0$ or 1 depending on the purpose of the sensitivity analysis (see subsection 2.5 ). The values of $q$ and $\theta$ at the boundary are computed by considering, as in the HLL formalism (40a)-(40b), that 
the boundary states are separated from the inside of the computational domain by a wave moving at speed $\lambda^{+}=u_{1}^{n}+c_{1}^{n}$. Then, equation (37b) is applicable with $h_{b}$ and $q_{b}$

$$
\begin{aligned}
& q_{1 / 2}^{n+1 / 2}=q_{1}^{n}+\left(h_{b}-h_{i}^{n}\right) \lambda^{+} \\
& \theta_{1 / 2}^{n+1 / 2}=\theta_{1}^{n}+\left(\eta_{b}-\eta_{i}^{n}\right) \lambda^{+}
\end{aligned}
$$

The water depth $h_{1 / 2}^{n+1 / 2}$ and the unit discharge $q_{1 / 2}^{n+1 / 2}$ as well as their sensitivity, being entirely determined at the interface $1 / 2$, the calculation of the momentum flux $\left(F_{2}\right)_{1 / 2}^{n+1 / 2}$ and the sensitivity flux $\left(G_{2}\right)_{1 / 2}^{n+1 / 2}$ is straightforward.

2. Prescribed unit discharge. The boundary conditions are

$$
\begin{gathered}
q_{1 / 2}^{n+1 / 2}=q_{b} \\
\theta_{1 / 2}^{n+1 / 2}=\theta_{b}=0 \text { or } 1
\end{gathered}
$$

Applying Eq. (37b) to the second components of $\mathbf{U}$ and s yields directly the second component of the fluxes for the flow variable and sensitivity

$$
\begin{aligned}
& \left(F_{2}\right)_{1 / 2}^{n+1 / 2}=\left(F_{2}\right)_{1}^{n}+\left(q_{b}-q_{i}^{n}\right) \lambda^{+} \\
& \left(G_{2}\right)_{1 / 2}^{n+1 / 2}=\left(G_{2}\right)_{1}^{n}+\left(\theta_{b}-\theta_{i}^{n}\right) \lambda^{+}
\end{aligned}
$$

3. Prescribed stage-discharge relationship. In the case of a known, nonlinear relationship between the water depth and the unit discharge, it is more convenient to rewrite (26) in the form of a relationship between the flow velocity $u$ and the wave celerity in still water $c$

$$
\begin{gathered}
f(u, c, \psi)=0 \\
\frac{\partial f}{\partial u} \nu+\frac{\partial f}{\partial c} \chi+\frac{\partial f}{\partial \psi}=0
\end{gathered}
$$

where $\chi$, and $\nu$ are respectively the sensitivity of $c$ and $u$. This set of equations is complemented by the Riemann invariants $u-2 c$ and $\nu-2 \chi$ for the flow and sensitivity respectively

$$
\begin{aligned}
& u_{1 / 2}^{n+1 / 2}-2 c_{1 / 2}^{n+1 / 2}=u_{1}^{n}-2 c_{1}^{n} \\
& \nu_{1 / 2}^{n+1 / 2}-2 \chi_{1 / 2}^{n+1 / 2}=\nu_{1}^{n}-2 \chi_{1}^{n}
\end{aligned}
$$

The systems $(71 \mathrm{a}, 72 \mathrm{a})$ and $(71 \mathrm{~b}, 72 \mathrm{~b})$ may be solved using any iterative procedure such as the NewtonRaphson technique.

\subsubsection{Transport equation}

Concerning the boundary condition for the variable subjected to passive transport, a value $r_{b}$ must be prescribed at the left-hand boundary if the unit-discharge $q$ is positive

$$
\begin{aligned}
& r_{1 / 2}^{n+1 / 2}=r_{b} \\
& \rho_{1 / 2}^{n+1 / 2}=\rho_{b}
\end{aligned}
$$

with $\rho_{b}=0$ or 1 depending on the purpose of the sensitivity analysis.

When the unit discharge is negative, the values of $r$ and $\rho$ inside the domain are conserved

$$
\begin{aligned}
& r_{1 / 2}^{n+1 / 2}=r_{1}^{n} \\
& \rho_{1 / 2}^{n+1 / 2}=\rho_{1}^{n}
\end{aligned}
$$

The calculation of the third components of $\mathbf{F}$ and $\mathbf{G}$ is then straightforward. 


\section{Computational examples}

\subsection{Sensitivity to the friction coefficient: steady flow on a sloping bed}

The purpose of this test is to assess the efficiency of the method for the sensitivity to the Manning's coefficient, in a case where a reference solution can be computed by discretizing the backwater curve equation

$$
\begin{gathered}
\frac{\mathrm{d} h}{\mathrm{~d} x}=\frac{S_{0}-S_{f}}{1-\mathrm{Fr}^{2}} \\
S_{f}=q^{2} n_{M}^{2} h^{-10 / 3}
\end{gathered}
$$

using the explicit Euler method. The boundary conditions are a prescribed unit-discharge $\left(q_{b}\right)$ upstream and a prescribed water depth $\left(h_{b}\right)$ downstream.

Since the flow is in steady state, the corresponding sensitivity equation is given by

$$
\begin{aligned}
\frac{\mathrm{d} \eta}{\mathrm{d} x} & =\eta \frac{\partial}{\partial h}\left(\frac{S_{0}-S_{f}}{1-\mathrm{Fr}^{2}}\right)+\varepsilon_{n_{M}} \frac{\partial}{\partial n_{M}}\left(\frac{S_{0}-S_{f}}{1-\mathrm{Fr}^{2}}\right) \\
& =\frac{1}{1-\mathrm{Fr}^{2}}\left[\left(\frac{10}{3} S_{f}-3 \mathrm{Fr}^{2} \frac{S_{0}-S_{f}}{1-\mathrm{Fr}^{2}}\right) \frac{\eta}{h}-2 \frac{S_{f}}{n_{M}} \varepsilon_{n_{M}}\right]
\end{aligned}
$$

with

$$
\begin{gathered}
\varepsilon_{n_{M}}(x)=1 \forall x \\
\eta_{b}(L)=0 \\
\theta(x)=0 \forall x
\end{gathered}
$$

and can also be discretized using the explicit Euler method.

The parameters of the test case are given in Table 1. The computed water depth, unit-discharge and their sensitivities to Manning's friction coefficient are given in Figure 1 for the proposed solver and the Euler approach. The empirical sensitivity profiles also given in Figure 1 are defined by the normalised difference between two simulations obtained using two slightly different values for $n_{M}$ (in this case: $0.025 \mathrm{~m}^{-1 / 3} \mathrm{~s} \mathrm{and}$ $0.024 \mathrm{~m}^{-1 / 3} \mathrm{~s}$ ). The physical interpretation of the sensitivity $\eta$ of $h$ with respect to $n_{M}$ (Figure $1 \mathrm{~b}$ ) is that a variation of 1 in $n_{M}$ leads to a variation in $h$ of about 21 upstream. Indeed, the calculation with $n_{M}=0.024$ gives $h=0.8892$ whereas the calculation with $n_{M}=0.025$ gives $h=0.8685$ (i.e. a difference of 0.021 ). The sensitivity of $h$ downstream remains nil because the water depth is prescribed at the downstream boundary. Since the flow is permanent and the unit-discharge is prescribed upstream, $q$ should remain constant in the whole domain and $\theta$ should be nil. The differences observed for the unit-discharge $q$ and its sensitivity $\theta$ near the downstream boundary are due to a difficulty of the proposed solver to assess the momentum values at cells, given boundary conditions at interfaces. The problem stems from the balancing process between the flux calculation at the interface and the values at cells ${ }^{1}$. Indeed, the values of $q$ and $\theta$ at the interfaces, i.e. the first component of the fluxes $\mathbf{F}$ and $\mathbf{G}$ respectively, are correctly estimated and are respectively equal to the prescribed value $q_{b}$ and to 0 in the whole domain (see Figure 2). Moreover, in this case, despite an unsatisfying value of $q$, its sensitivity $\theta$ is better estimated by the direct approach than by the empirical method.

To assess the convergence of the proposed approach, a convergence analysis is performed in the uniform case, for which an analytical solution is known. The uniform flow is obtained by prescribing $h_{b}$ to be the normal height

$$
h_{b}=h_{N}=\left(\frac{q n_{M}}{\sqrt{S_{0}}}\right)^{3 / 5}
$$

In this configuration, Eq. (76) is simplified into

$$
\frac{\mathrm{d} \eta}{\mathrm{d} x}=\frac{S_{f}}{1-\mathrm{Fr}^{2}}\left[\frac{10}{3} \frac{\eta}{h}-2 \frac{\varepsilon_{n_{M}}}{n_{M}}\right]
$$

This first order differential equation admits the following analytical solution

$$
\eta(x)=\frac{3}{5} \frac{h_{N}}{n_{M}}\left(1-e^{-\frac{S_{f}}{1-\mathrm{Fr}^{2}} \cdot \frac{10}{3 h} L}\right) e^{\frac{S_{f}}{1-\mathrm{Fr}^{2}} \cdot \frac{10}{3 h} x}
$$

\footnotetext{
${ }^{1}$ Note that the isolated points in Figure 1c and 1d are not artefacts due to the drawing of only one point every 25.
} 


\begin{tabular}{lll}
\hline Symbol & Meaning & Value \\
\hline$g$ & Gravitational acceleration & $9.81 \mathrm{~m} \mathrm{~s}^{-2}$ \\
$L$ & Length of the simulation domain & $3000 \mathrm{~m}$ \\
$q_{b}$ & Unit-discharge prescribed at the upstream boundary & $1 \mathrm{~m}^{2} \mathrm{~s}^{-1}$ \\
$h_{b}$ & Water depth prescribed at the downstream boundary & $0.8 \mathrm{~m}$ \\
$S_{0}$ & Bottom slope & $1 \cdot 10^{-3}$ \\
$h_{0}$ & Initial water depth in the domain & $1 \mathrm{~m}$ \\
$q_{0}$ & Initial unit-discharge in the domain & $1 \mathrm{~m}^{2} \mathrm{~s}^{-1}$ \\
$n_{M}$ & Manning's friction coefficient & $0.025 \mathrm{~m}^{-1 / 3} \mathrm{~s}$ \\
$(\Delta x)_{r}$ & Cells width used for the reference solution & $0.001 \mathrm{~m}$ \\
$(\Delta x)_{n}$ & Cells width used for the numerical solution & $1 \mathrm{~m}$ \\
$T$ & Computational time & $15000 \mathrm{~s}$ \\
\hline
\end{tabular}

Table 1: Steady flow on a sloping bed: problem parameters.

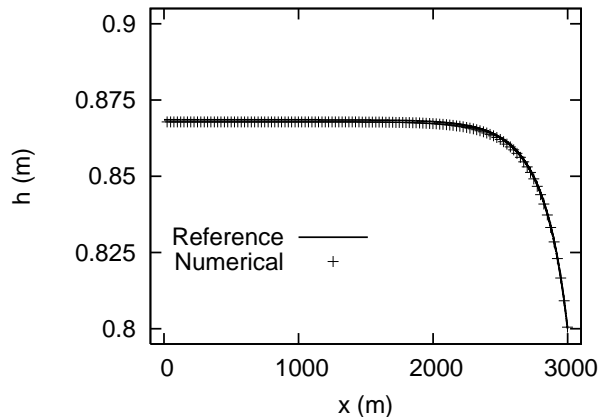

(a)

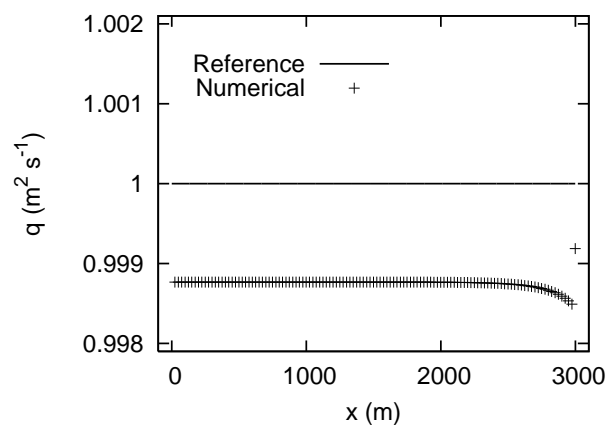

(c)

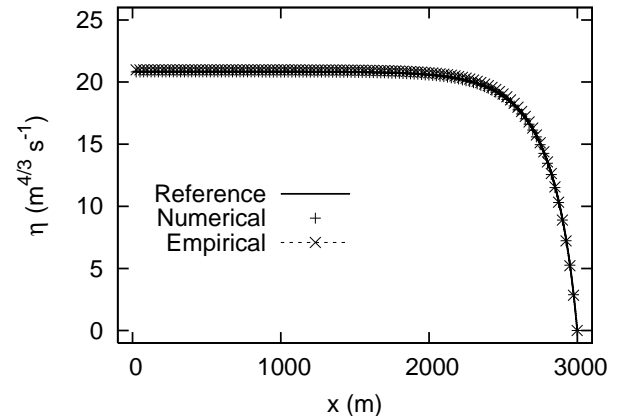

(b)

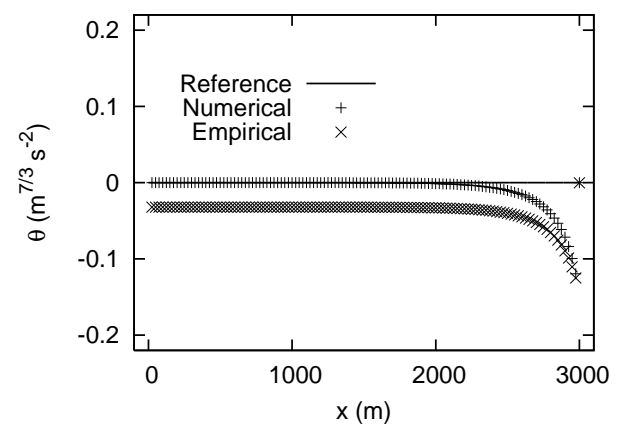

(d)

Figure 1: Sensitivity to the Manning coefficient for a steady state flow on a sloping bed. Comparison between the proposed solver and the explicit Euler method (reference). For the sake of clarity, only one point every 25 is represented. 


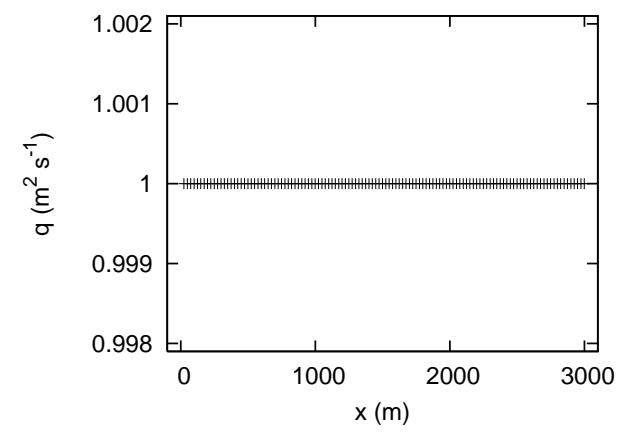

(a)

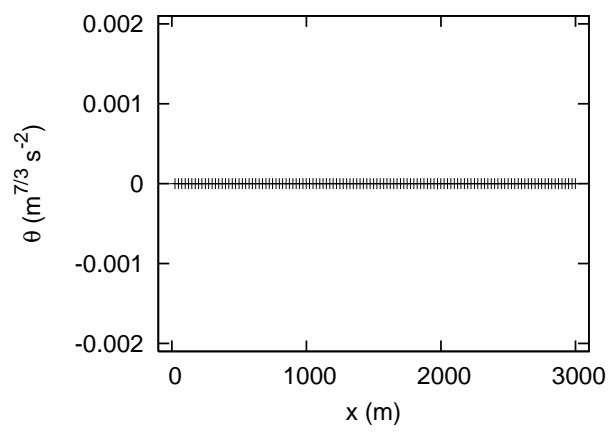

(b)

Figure 2: Sensitivity to the Manning coefficient for a steady state flow on a sloping bed. Values of $q$ and $\theta$ computed at the interfaces.

The convergence analysis is performed using the parameters given in Table 1 , except $h_{b}=h_{N} \approx 0.8685 \mathrm{~m}$, and eight different values of the spatial discretization step: $\Delta x=\{5 ; 3 ; 2 ; 1 ; 0.5 ; 0.25 ; 0.1 ; 0.05\} \mathrm{m}$. The corresponding values of $\Delta t_{\max }$ are automatically computed using Eq. (54). The variations $L_{1^{-}}$and $L_{2}$-norms of the difference between the analytical and numerical solutions with the cell width $\Delta x$ are represented in Figure 3 for the variables $h, q$ and their sensitivities. The $L_{p}$-norm $(p=1,2)$ is defined as:

$$
L_{p}=\left\{\sum_{i}\left[Y_{i}-Y\left(x_{i}\right)\right]^{p} \Delta x_{i}\right\}^{1 / p}
$$

where $Y_{i}$ and $Y\left(x_{i}\right)$ represent respectively the numerical and analytical solutions with $Y$ being either $h, q$, $\eta$ or $\theta$.

Both norms decrease with $\Delta x$ - the $L_{2}$-norm with a slope about twice the one of the $L_{1}$-norm - indicating a uniform convergence of the numerical solution to the analytical solution.

\subsection{Sensitivity to the initial conditions: dam-break on a sloping bed}

The dam-break problem is an initial value problem in which the water is initially at rest and the water levels are different on both sides of the dam. The variable subjected to passive scalar transport can also have different values on both sides of the dam. This problem is thus a Riemann problem defined as

$$
\begin{gathered}
h(x, 0)= \begin{cases}h_{\mathrm{L}} & \text { for } x<x_{0} \\
h_{\mathrm{R}} & \text { for } x>x_{0}\end{cases} \\
q(x, 0)=0 \\
r(x, 0)= \begin{cases}r_{\mathrm{L}} & \text { for } x<x_{0} \\
r_{\mathrm{R}} & \text { for } x>x_{0}\end{cases}
\end{gathered}
$$

Note that this initial condition does not correspond to equilibrium conditions but gives the advantage that the analytical solution is easy to compute (see [7] for more details).

A sensitivity analysis with respect to the initial water depth $h_{\mathrm{L}}$ in the dam is performed. The analytical, empirical and numerical solutions are plotted in Figure 4 for a positive slope and in Figure 5 for a negative slope with the parameters given in Table 2. The higher the magnitude of the sensitivity, the more sensitive the variable with respect to the parameter. For example in Figure $4 \mathrm{e}$, the value of $\theta=\partial q / \partial h_{\mathrm{L}}$ for $x=0$ is 1.96; this means that a variation of 1 for $h_{\mathrm{L}}$ leads to a variation of approximatively 1.96 for $q$ (note that this approximation remains valid for small variations). Indeed, the calculation using $h_{\mathrm{L}}=10$ gives $q_{x=0}=19.62$ whereas the calculation using $h_{\mathrm{L}}=11$ gives $q_{x=0}=21.58$ (i.e a difference of 1.96).

The empirical sensitivity profiles have been obtained using two slightly different values for $h_{\mathrm{L}}$ (i.e. $10 \mathrm{~m}$ and $10.01 \mathrm{~m}$ ) and for $v_{\mathrm{L}}$ (i.e. 1 and 1.01, the unit of which depends on the nature of the variable). In Figures 4 and 5 the same scale is used to plot the empirical and numerical sensitivity profiles, but the empirical profiles exhibit artificial peaks in the neighbourhood of the shock that exceed by far the scale range. This is due to the fact that the shock celerity is not exactly the same between both simulations (because it depends on 


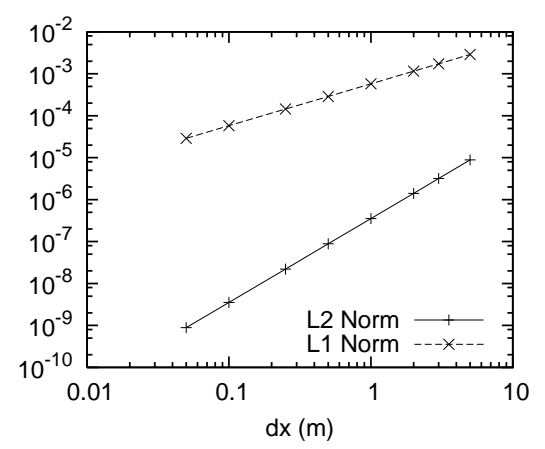

(a) $h$

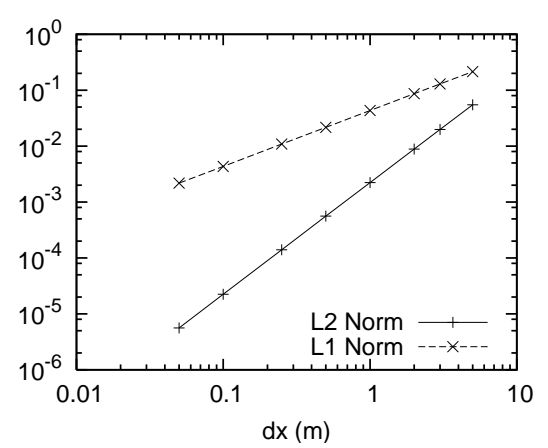

(c) $\eta$

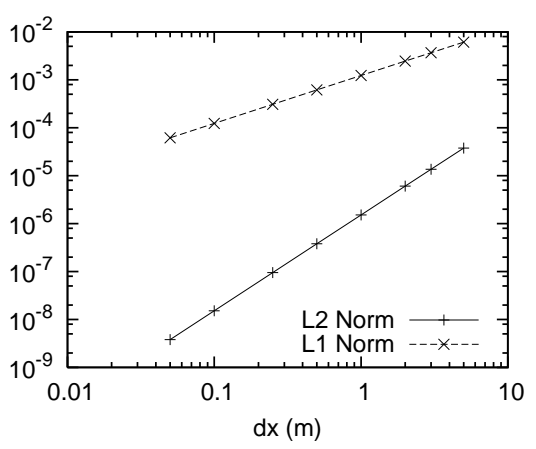

(b) $q$

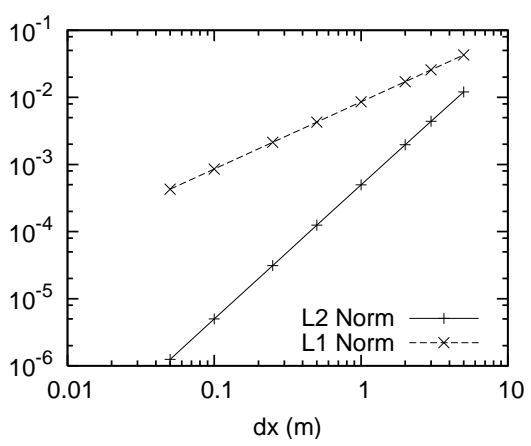

(d) $\theta$

Figure 3: Convergence analysis. $L_{1}$ and $L_{2}$ norms of the error as functions of cell size (logarithmic scale).

the value of $h_{\mathrm{L}}$ ). This behaviour is eliminated with the proposed solver, thanks to the introduction of the Dirac-source term across the shock.

\subsection{Sensitivity to the boundary conditions: bore propagation into a frictionless channel}

The purpose of this test is to assess the accuracy of the proposed discretization for the sensitivity to boundary conditions in the presence of discontinuous solutions. Consider a horizontal, frictionless channel where the water is initially at rest, with a constant initial depth $h_{0}$. At $t=0$, the unit discharge and the value of the variable subjected to passive transport, at the channel upstream $(x=0)$, change instantaneously from 0 to constant values: $q_{b}$ and $v_{b}$. A bore appears and propagates into the channel at a constant speed $c_{s}$. Writing the jump relationships across the bore leads to

$$
\begin{gathered}
q_{b}=\left(h_{b}-h_{0}\right) c_{s} \\
\frac{q_{b}^{2}}{h_{b}}+\frac{g}{2}\left(h_{b}^{2}-h_{0}^{2}\right)=q_{b} c_{s}
\end{gathered}
$$

where $h_{b}$ is the water depth at the boundary. Eqs (83) are solved uniquely for $h_{b}$ and $c_{s}$ using iterative linearization techniques such as Newton-Raphson's method. Since the purpose is to study the sensitivity of the solution to the values of $q_{b}$ and $v_{b}$, the sensitivity boundary condition are $\theta_{b}=1$ and $\omega_{b}=1$. The jump relationships for the sensitivity are obtained by differentiating (83) with respect to $q_{b}$, and noticing that the initial water depth sensitivity $\eta_{0}$ in the channel is zero

$$
\begin{gathered}
\eta_{b} c_{s}+\left(h_{b}-h_{0}\right) \frac{\partial c_{s}}{\partial q_{b}}=1 \\
2 u_{b}-u_{b}^{2} \eta_{b}+g h_{b} \eta_{b}=c_{s}+q_{b} \frac{\partial c_{s}}{\partial q_{b}}
\end{gathered}
$$

Eqs (84) can be solved uniquely for $\eta_{b}$ and $\partial c_{s} / \partial q_{b}$. The bore concerning the variable subjected to passive transport propagates at the constant speed of the contact wave: $\lambda^{(2)}=u=u_{b}$.

The set of parameters used for this application example is given in Table 3. The empirical sensitivity profiles have been obtained using two slightly different values for $q_{b}$ (i.e. $1 \mathrm{~m}^{2} \mathrm{~s}^{-1}$ and $1.01 \mathrm{~m}^{2} \mathrm{~s}^{-1}$ ) and for 


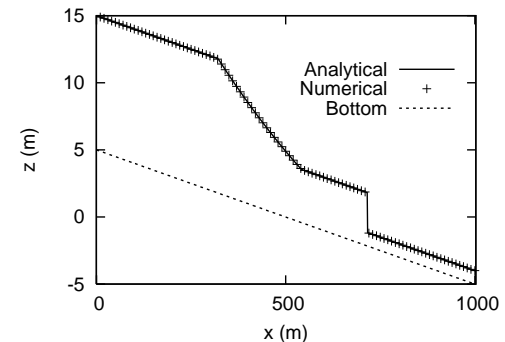

(a)

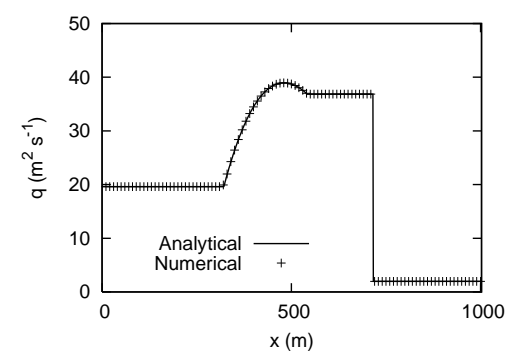

(d)

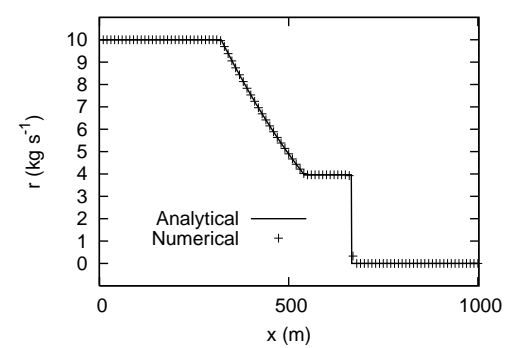

(g)

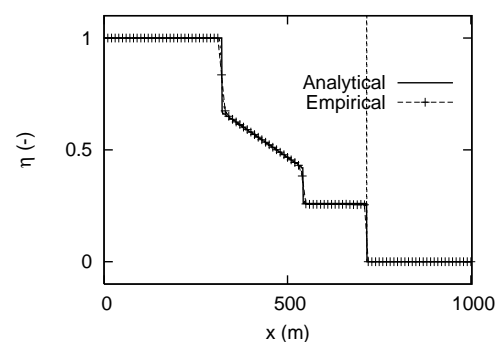

(b)

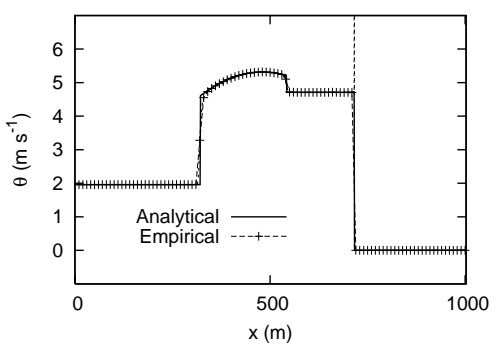

(e)

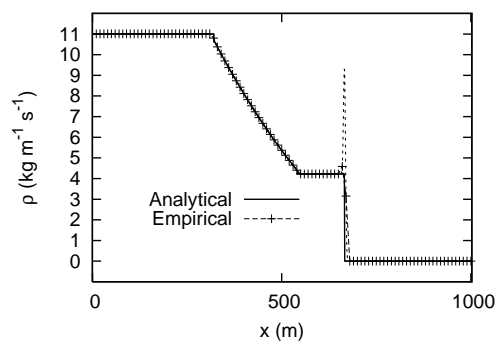

(h)

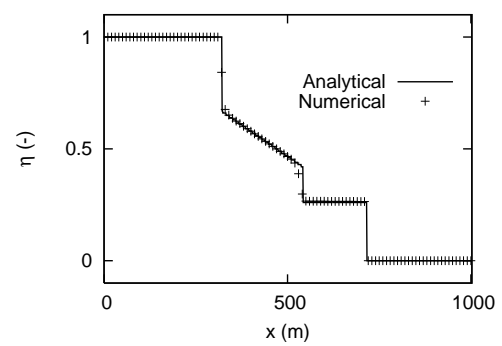

(c)

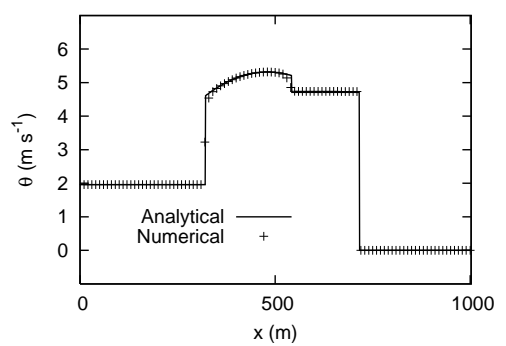

(f)

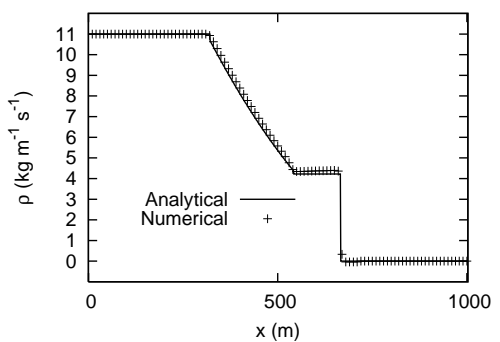

(i)

Figure 4: Dam-break problem on a positive slope. Left: flow solution, middle: empirical sensitivity, right: numerical sensitivity. For the sake of clarity, only one point every 100 is represented. The values reached by the peaks in subfigures (b) and (e) are respectively about 175 and 2130. 


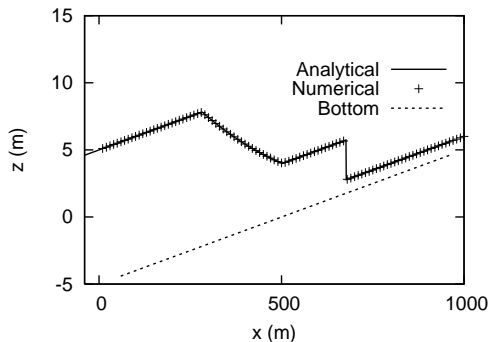

(a)

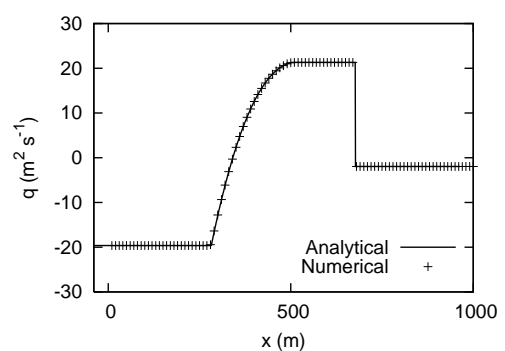

(d)

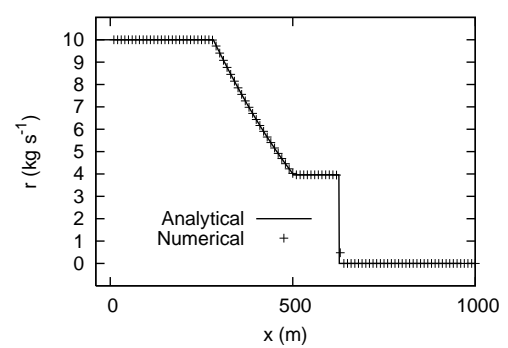

(g)

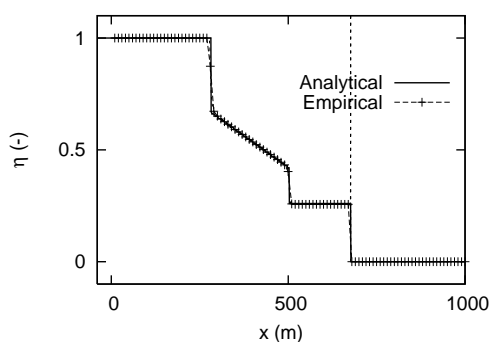

(b)

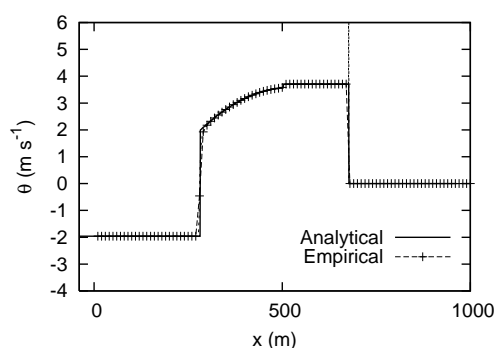

(e)

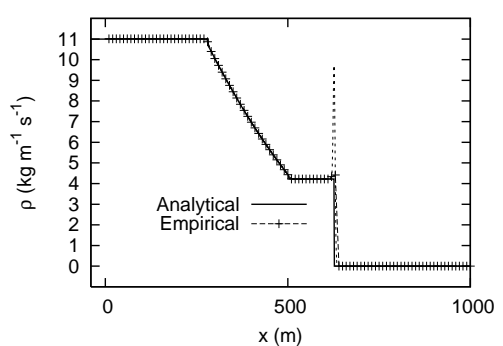

(h)

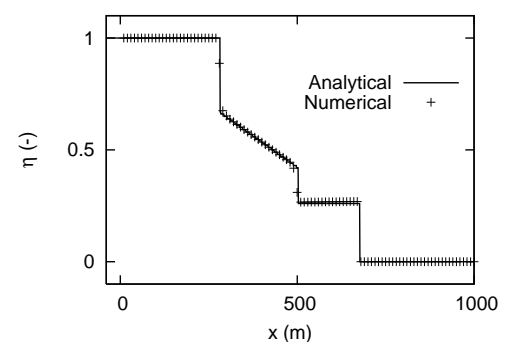

(c)

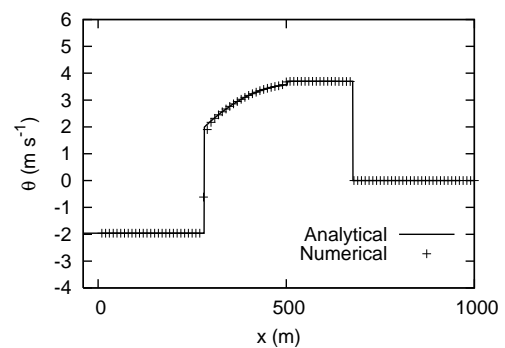

(f)

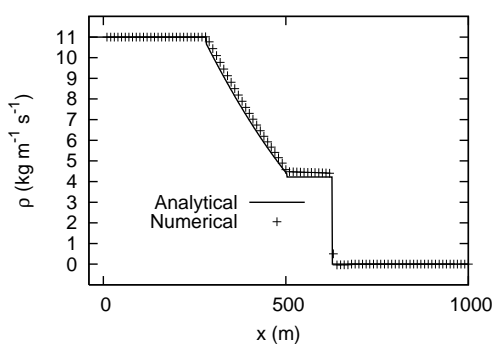

(i)

Figure 5: Dam-break problem on a negative slope. Left: flow solution, middle: empirical sensitivity, right: numerical sensitivity. The values reached by the peaks in subfigures (b) and (e) are respectively about 170 and 1245 . 


\begin{tabular}{|c|c|c|}
\hline Symbol & Meaning & Value \\
\hline$g$ & Gravitational acceleration & $9.81 \mathrm{~m} \mathrm{~s}^{-2}$ \\
\hline$L$ & Length of the simulation domain & $1000 \mathrm{~m}$ \\
\hline$S_{0}$ & Bottom slope & 0.01 and -0.01 \\
\hline$h_{\mathrm{L}}$ & Water depth on the left-hand side of the initial discontinuity & $10 \mathrm{~m}$ \\
\hline$h_{\mathrm{R}}$ & Water depth on the right-hand side of the initial discontinuity & $1 \mathrm{~m}$ \\
\hline$q_{\mathrm{L}}$ and $q_{\mathrm{R}}$ & $x$ unit discharge on the left- and right-hand sides of the initial discontinuity & $0 \mathrm{~m}^{2} \mathrm{~s}$ \\
\hline & $\begin{array}{l}\text { initial value of the variable subjected to passive transport on the left-hand } \\
\text { side of the discontinuity }\end{array}$ & $1 \mathrm{~kg} \mathrm{~s}^{-1}$ \\
\hline$v_{\mathrm{R}}$ & $\begin{array}{l}\text { initial value of the variable subjected to passive transport on the right-hand } \\
\text { side of the discontinuity }\end{array}$ & $0 \mathrm{~kg} \mathrm{~s}^{-1}$ \\
\hline$x_{0}$ & Abscissa of the initial discontinuity & $500 \mathrm{~m}$ \\
\hline$\eta_{\mathrm{L}}$ & Water depth sensitivity on the left-hand side of the initial discontinuity & $1(-)$ \\
\hline$\eta_{\mathrm{R}}$ & Water depth sensitivity on the right-hand side of the initial discontinuity & $0(-)$ \\
\hline$\theta_{\mathrm{L}}$ and $\theta_{\mathrm{R}}$ & $\begin{array}{l}\text { Sensitivity of the } x \text { unit discharge on the left- and right-hand sides of the } \\
\text { initial discontinuity }\end{array}$ & $0 \mathrm{~ms}^{-1}$ \\
\hline$\omega_{\mathrm{L}}$ & $\begin{array}{l}\text { Sensitivity of the variable subjected to passive transport on the left-hand side } \\
\text { of the initial discontinuity }\end{array}$ & $1 \mathrm{~kg} \mathrm{~m}^{-1} \mathrm{~s}^{-1}$ \\
\hline$\omega_{\mathrm{R}}$ & $\begin{array}{l}\text { Sensitivity of the variable subjected to passive transport on the right-hand } \\
\text { side of the initial discontinuity }\end{array}$ & $0 \mathrm{~kg} \mathrm{~m}^{-1} \mathrm{~s}^{-1}$ \\
\hline$n_{M}$ & Manning's friction coefficient & $0 . \mathrm{m}^{-1 / 3} \mathrm{~s}$ \\
\hline$\Delta x$ & Cells width & $0.1 \mathrm{~m}$ \\
\hline$T$ & Computational time & $20 \mathrm{~s}$ \\
\hline
\end{tabular}

Table 2: Dam-break on a sloping bed: problem parameters.

\begin{tabular}{lll}
\hline Symbol & Meaning & Value \\
\hline$g$ & Gravitational acceleration & $9.81 \mathrm{~m} \mathrm{~s}^{-2}$ \\
$L$ & Length of the simulation domain & $100 \mathrm{~m}$ \\
$\Delta x$ & Cells width & $1 \mathrm{~m}$ \\
$t$ & Computational time & $15 \mathrm{~s}$ \\
$h_{0}$ & Initial water depth & $1 \mathrm{~m}$ \\
$q_{b}$ & Unit-discharge prescribed at the upstream boundary & $1 \mathrm{~m}^{2} \mathrm{~s}^{-1}$ \\
$\theta_{b}$ & Upstream discharge sensitivity & $1(-)$ \\
$v_{b}$ & Value prescribed at the upstream boundary, for the variable subjected to & $1 \mathrm{~kg} \mathrm{~s}^{-1}$ \\
& passive transport & $1 \mathrm{~kg} \mathrm{~m}^{-2} \mathrm{~s}^{-2}$ \\
$\omega_{b}$ & Upstream sensitivity for the variable subjected to passive transport & \\
\hline
\end{tabular}

Table 3: Bore propagation into a frictionless channel: problem parameters. All initial conditions but $h_{0}$ are nil.

$v_{\mathrm{L}}$ (i.e. 1 and 1.01, the unit of which depends on the nature of the variable). The analytical, numerical, and empirical sensitivity profiles are given in Figure 6. As shown in the previous test case, the empirical sensitivity profiles also exhibit artificial peaks in the neighbourhood of the shock, that are not present with the proposed solver. In this case, however, diffusion of the numerical solution can be observed near the shock for $r$ and $\rho$ (Figures $6 \mathrm{~g}$ and $6 \mathrm{~h}$ ) that may indicate the need for a higher order reconstruction.

\subsection{Application to a non rectangular river profile}

The direct sensitivity approach can be applied to non rectangular river profiles. An example is given here for a trapezoidal channel, where the cross-sectional area $A$ is defined by

$$
A=\left(B_{0}+h \tan \alpha\right) h
$$

where $B_{0}$ is the bottom width, $h$ the water depth and $\alpha$ the angle formed by the channel sides to the vertical. The governing equations (1) and (2) can be rewritten as

$$
\frac{\partial \mathbf{U}_{\mathbf{T}}}{\partial t}+\frac{\partial \mathbf{F}_{\mathbf{T}}}{\partial x}=\mathbf{S}_{\mathbf{T}}
$$




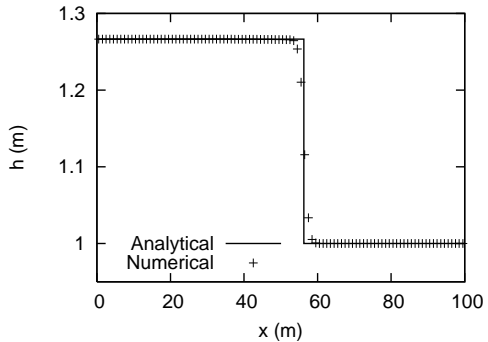

(a)

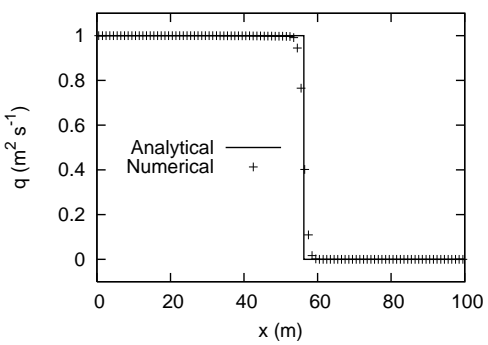

(d)

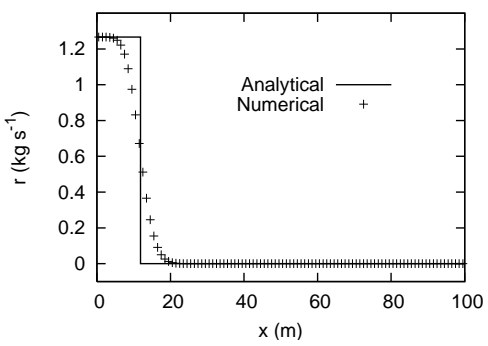

(g)

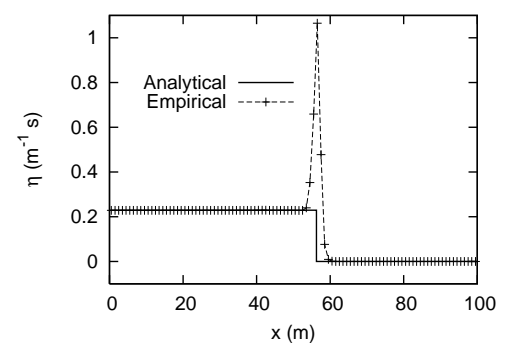

(b)

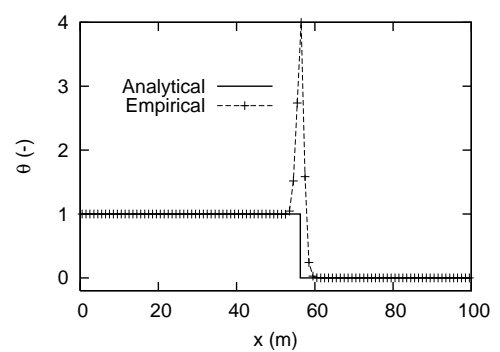

(e)

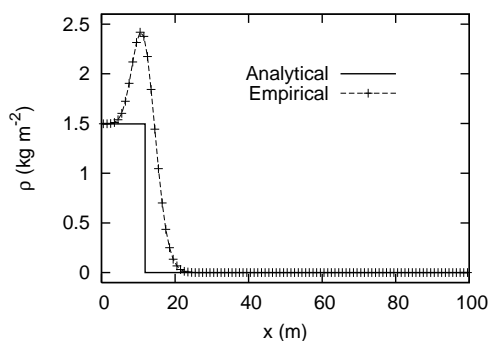

(h)

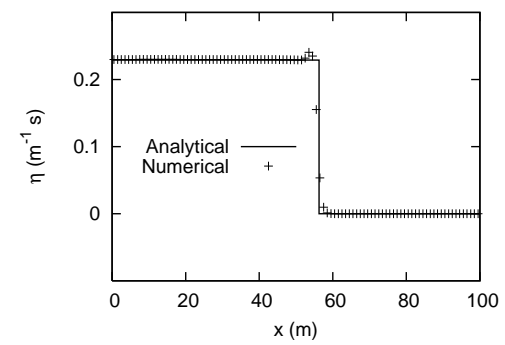

(c)

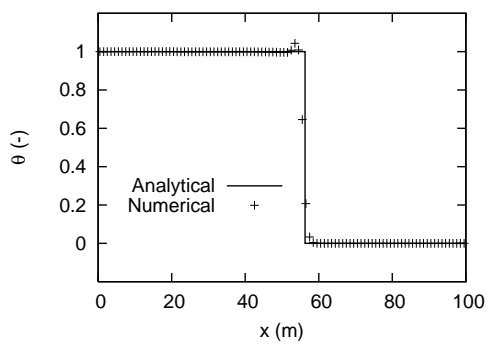

(f)

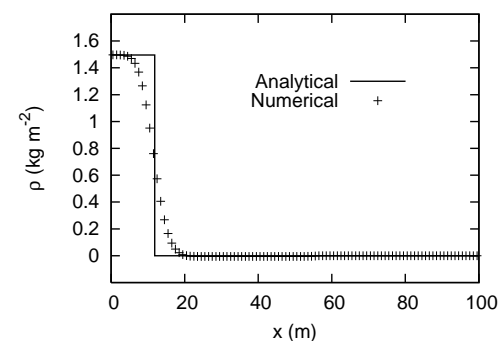

(i)

Figure 6: Bore propagation into a frictionless channel and sensitivity with respect to the prescribed unit discharge $q_{b}$. Left: flow solution, middle: empirical sensitivity, right: numerical sensitivity. 


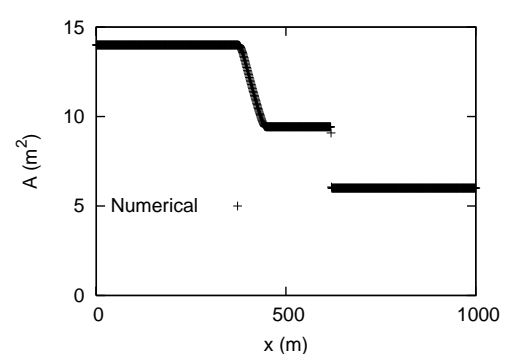

(a)

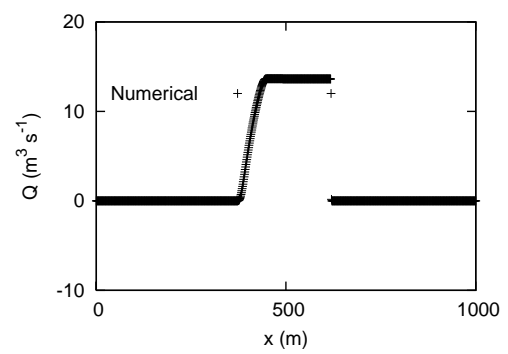

(c)

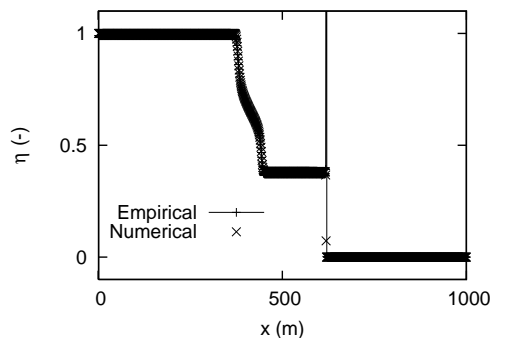

(b)

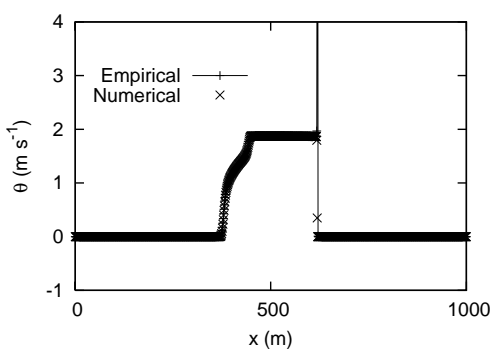

(d)

Figure 7: Dambreak problem for a trapezoidal channel. Left: flow solution, right: empirical and numerical sensitivities.

$$
\mathbf{U}_{\mathbf{T}}=\left(\begin{array}{c}
A \\
Q
\end{array}\right), \mathbf{F}_{\mathbf{T}}=\left(\begin{array}{c}
Q \\
Q^{2} / A+g h^{2} B_{0} / 2+g h^{3} \tan \alpha / 3
\end{array}\right), \mathbf{S}_{\mathbf{T}}=\left(\begin{array}{c}
0 \\
g A\left(S_{0}-S_{f}\right)
\end{array}\right)
$$

In this case, the expression of the sensitivity flux becomes

$$
\mathbf{G}_{\mathbf{T}}=\left(\begin{array}{c}
\theta \\
g A\left(B_{0}^{2}+4 A \tan \alpha\right)^{1 / 2} \eta-u^{2} \eta+2 u \theta
\end{array}\right)
$$

The flow solution of the dam-break problem applied to a trapezoidal channel is plotted in Figure 7(a) and 7(c) with the parameters given in Table 4 . The sensitivity of $A$ and $Q$ with respect to the initial crosssectional area in the dam, is plotted in Figure 7(b) and 7(d). In this case again, the direct computation of the sensitivity removes the artefacts of the empirical method.

\section{Conclusion}

The sensitivity equations were derived for the one-dimensional Shallow Water Equations with scalar transport and source terms. A finite volume-based numerical technique was proposed for both continuous and discontinuous flow solutions. In this latter case a Dirac-source term, introduced across shocks in the governing sensitivity equations, increases complexity in the solution of the sensitivity problem. However, this approach enables elimination of numerical artifacts present in the empirical method, such as infinite sensitivity values at discontinuities.

Ongoing research focuses on the generalization of the present technique to the solution of the twodimensional shallow water equations on unstructured grids. As shown in [5], such a generalization is not trivial because solving the two-dimensional shallow water equations involves solving a third conservation equation for transverse momentum. In the present work, to preserve the invariance property of the sensitivity Riemann invariant along the contact wave, a part of the third equation has been discretized in non-conservation form. In two dimensions, not discretizing the transverse momentum equation (that can be considered as a passive tracer) in a fully conservative manner can be expected to yield accuracy and/or stability problems. Moreover, the determination of the exact location of shocks in two dimensions will also increase the complexity of the problem.

The difficulty of the proposed solver in dealing with the imposed boundary conditions in the steady state configuration with bottom slope and friction (section 4.1), stems from the HLLC solver itself, in the balancing process between the flux calculation at the interface and the values at cells. The development of sensitivity equations for the approximate state Riemann solver presented in [12] is thus under investigation. Since using 


\begin{tabular}{|c|c|c|}
\hline Symbol & Meaning & Value \\
\hline$g$ & Gravitational acceleration & $9.81 \mathrm{~m} \mathrm{~s}^{-2}$ \\
\hline$L$ & Length of the simulation domain & $1000 \mathrm{~m}$ \\
\hline$B_{0}$ & Bottom width of the channel & $5 \mathrm{~m}$ \\
\hline$\alpha$ & Angle between channel sides and the vertical & $\pi / 4$ \\
\hline$S_{0}$ & Bottom slope & 0. \\
\hline$h_{\mathrm{L}}$ & Water depth on the left-hand side of the initial discontinuity & $2 \mathrm{~m}$ \\
\hline$h_{\mathrm{R}}$ & Water depth on the right-hand side of the initial discontinuity & $1 \mathrm{~m}$ \\
\hline$Q_{\mathrm{L}}$ and $Q_{\mathrm{R}}$ & Discharge on the left- and right-hand sides of the initial discontinuity & $0 \mathrm{~m}^{2} \mathrm{~s}$ \\
\hline$x_{0}$ & Abscissa of the initial discontinuity & $500 \mathrm{~m}$ \\
\hline$\eta_{\mathrm{L}}$ & $\begin{array}{l}\text { Sensitivity of the water cross-sectional area on the left-hand side of the initial } \\
\text { discontinuity }\end{array}$ & $1(-)$ \\
\hline$\eta_{\mathrm{R}}$ & $\begin{array}{l}\text { Sensitivity of the water cross-sectional area on the right-hand side of the } \\
\text { initial discontinuity }\end{array}$ & $0(-)$ \\
\hline$\theta_{\mathrm{L}}$ and $\theta_{\mathrm{R}}$ & $\begin{array}{l}\text { Sensitivity of the discharge on the left- and right-hand sides of the initial } \\
\text { discontinuity }\end{array}$ & $0 \mathrm{~ms}^{-1}$ \\
\hline$n_{M}$ & Manning's friction coefficient & $0 . \mathrm{m}^{-1 / 3} \mathrm{~s}$ \\
\hline$\Delta x$ & Cells width & $0.1 \mathrm{~m}$ \\
\hline$T$ & Computational time & $30 \mathrm{~s}$ \\
\hline
\end{tabular}

Table 4: Dam-break on a trapezoidal channel: problem parameters.

higher-order schemes for scalar sensitivity equations has been shown to improve the accuracy of sensitivity solutions in a number of cases [8], the applicability of fast, higher-order numerical reconstruction techniques such as the MUSCL-EVR approach [16] is another path for research. The strong diffusion, observed for the variable subjected to passive scalar transport and for its sensitivity in the bore propagation problem, is expected to be limited using these techniques.

\section{Acknowledgements}

This work was supported by Research Contract ANR-05-PGCU-004, "RIVES" funded by the French Agence Nationale de la Recherche (ANR) / National Research Agency. The contribution and support of the following partners is gratefully acknowledged: Cemagref, CETE Méditerranée, CETMEF, LMFA UMR 5509 (CNRS, ECL, INSA Lyon, UCB), INSAVALOR, LCPC, LRPC Bordeaux, LRPC Clermont-Ferrand, Sogreah, URGC (INSA Lyon), UTC.

\section{References}

[1] C. Bardos and O. Pironneau. A formalism for the differentiation of conservation laws. Comptes Rendus de l'Académie des Sciences Paris, Ser. I, 335:839-845, 2002.

[2] J. A. Cunge, F. M. Jr Holly, and A. Verwey. Practical aspects of river computational hydraulics. Pitman Advanced Pub. Program, 1980.

[3] S. F. Davis. Simplified second-order Godunov-type methods. SIAM Journal on Scientific and Statistical Computing, 9:455-473, 1988.

[4] C. Delenne, V. Guinot, and B. Cappelaere. Direct sensitivity computation for the Saint-Venant equations with hydraulic jumps. Comptes Rendus Mécanique, 336:766-771, 2008.

[5] V. Guinot. Upwind finite volume solution of sensitivity equations for hyperbolic systems of conservation laws with discontinuous solutions. Computers and Fluids, 38:1697-1709, 2009.

[6] V. Guinot and B. Cappelaere. Sensitivity equations for the one-dimensional shallow water equations: Practical application to model calibration. Journal of Hydrologic Engineering, 14(8):858-861, 2009.

[7] V. Guinot, C. Delenne, and B. Cappelaere. An approximate-state Riemann solver for sensitivity equations with discontinuous solutions. Advances in Water Resources, 31(1):61-77, 2009.

[8] V. Guinot, M. Leménager, and B. Cappelaere. Sensitivity equations for hyperbolic conservation lawbased flow models. Advances in Water Resources, 30:1943-1961, 2007. 
[9] M. D. Gunzburger. Sensitivities, adjoints and flow optimization problems. International Journal for Numerical Methods in Fluids, 31:53-78, 1999.

[10] A. Harten, P.-D. Lax, and B. Van-Leer. On upstream differencing and Godunov-type schemes for hyperbolic conservation laws. SIAM Review, 25:35-61, 1983.

[11] P.-D. Lax. Hyperbolic systems of conservation laws. Communications on Pure and Applied Mathematics, 10:537-566, 1957.

[12] J. Lhomme and V. Guinot. A general approximate-state Riemann solver for hyperbolic systems of conservation laws with source terms. International Journal for Numerical Methods in Fluids, 53:15091540, 2007.

[13] S. Lu and P. Sagaut. Direct sensitivity analysis for smooth unsteady compressible flow using complex differentiation. International Journal for Numerical Methods in Fluids, 53(12):1863-1886, 2007.

[14] M. Nujíc. Efficient implementation of non-oscillatory schemes for the computation of free-surface flows. Jounal of Hydraulic Research, 33:101-111, 1995.

[15] A. Saltelli, S. Tarantola, and F. Campolongo. Sensitivity analysis in practice: a guide to assessing scientific models. Wiley, 2008.

[16] S. Soares Frazão and V. Guinot. An eigenvector-based reconstruction scheme for the shallow-water equations on two-dimensional unstructured meshes. International Journal for Numerical Methods in Fluids, 53:23-55, 2007.

[17] J. J. Stoker. Water Waves. Interscience, Wiley, New-York, 1957.

[18] E. F. Toro, M. Spruce, and W. Speares. Restoration of the contact surface in the HLL-Riemann solver. Shock Waves, 4:25-34, 1994.

[19] M. E. Vasquez-Cendon. Improved treatment of source terms in upwind schemes for the shallow water equations in channels with irregular geometry. Journal of Computational Physics, 148(2):497-526, 1999. 